\title{
On the gas temperature in the shocked circumstellar envelopes of pulsating stars
}

\section{Dynamical models for AGB star winds including time-dependent dust formation and non-LTE cooling}

\begin{abstract}
V. Schirrmacher, P. Woitke, and E. Sedlmayr
Zentrum für Astronomie und Astrophysik, Technische Universität Berlin, Hardenbergstraße 36, 10623 Berlin, Germany

Received 30 October 2002 / Accepted 18 March 2003

Abstract. In this paper, we examine the mass loss mechanism of C-rich AGB stars by means of spherically symmetric model calculations, which combine hydrodynamics, grey radiative transfer (with constant gas and variable dust opacity) and timedependent dust formation (based on equilibrium chemistry and modified classical nucleation theory) with a time-independent non-LTE description of the state functions of the gas, in particular concerning the radiative heating and cooling function. According to our models, the dissipative heating by shock waves created by the stellar pulsation does not lead to a long-lasting increase of the gas temperatures close to the star, because the radiative cooling is too effective. The gas results to be mostly in radiative equilibrium (RE), except for some narrow but hot post-shock cooling zones. Consequently, the dust formation and wind acceleration proceeds in a similar way as described by Fleischer et al. (1992) and we find a dust-driven wind triggered by the stellar pulsation, but no evidence for a purely pulsation-driven mass loss. Several new effects occur in the models which are causally connected with the non-LTE state functions. In particular, the dissociation/re-formation of $\mathrm{H}_{2}$ consumes/liberates so much energy that the radiative relaxation towards RE can be significantly delayed in regions where the phase transition $\mathrm{H} \rightleftharpoons \mathrm{H}_{2}$ takes place. These regions may stay in non-RE for a considerable fraction of the stellar pulsation period. The radiative cooling behind the strongest, dissociative shock waves $\left(\approx 20 \mathrm{~km} \mathrm{~s}^{-1}\right)$ usually proceeds in a two-step process where the initially rapid cooling by permitted atomic lines down to $\approx 6000 \mathrm{~K}$ is followed by a second phase of intense radiative cooling below $\approx 3000 \mathrm{~K}$, as soon as the first molecules (e.g. CO) have formed. In the meantime, the gas cools slowly by forbidden metal emission lines, or by adiabatic expansion. This re-increase of the radiative cooling function with decreasing gas temperature causes a radiative instability which may temporarily lead to a coexistence of cool molecule-rich and warm molecule-poor regions in the radiative relaxation zone.
\end{abstract}

Key words. equation of state - instabilities - shock waves - stars: AGB and post-AGB - mass loss - winds, outflows

\section{Introduction}

Intermediate and low-mass stars (with main sequence mass $\approx 1 \ldots 8 M_{\odot}$ ) cease their life on the asymptotic giant branch (AGB), where they lose a substantial fraction of their initial mass by stellar winds (Habing 1996; Wallerstein \& Knapp 1998). The mass loss of AGB stars finally occurs on time-scales shorter than typical nuclear burning timescales, which drives them towards the planetary-nebula phase (Wachter et al. 2002). The stellar outflows of AGB stars are furthermore important for the enrichment of the interstellar medium with dust and heavy elements. A detailed understanding of the dynamical processes responsible for the formation of AGB star winds is therefore essential not only for the modelling of late stages of stellar evolution, but also as a key ingredient for the chemical evolution of galaxies.

Send offprint requests to: V. Schirrmacher, e-mail: vasco@astro.physik.tu-berlin.de
The mass loss mechanism of AGB stars is still a matter of debate (see e.g. Woitke 2002). Radiation pressure on dust grains provides a satisfactory explanation for very cool and luminous AGB stars (e.g. Gail \& Sedlmayr 1987a). However, for average AGB stellar parameters, dust-driven winds already require the assistance of another mechanism which supplies the dust formation zone with enough stellar matter, most likely the stellar pulsation (Fleischer et al. 1992, 1995; Höfner et al. 1995; Winters et al. 2000). For higher effective temperatures $\left(T_{\text {eff }} \gtrsim 3200 \mathrm{~K}\right)$, the dust-driving becomes finally ineffective because the dust formation zone is located too far away from the stellar surface. Furthermore, concerning metalpoor AGB stars like in the LMC, the maximum radiation pressure on dust grains attainable by condensing out all available heavy elements, may not be sufficient to drive a stellar wind (Helling et al. 2002). Thus, the concept of dust-driven winds is probably not applicable on the complete AGB. Other mechanisms are important to understand the mass loss of these stars, 
e.g. radiation pressure on molecules (Maciel 1977; Helling et al. 2000), the momentum input by small-scale magnetohydrodynamic or acoustic waves (Pijpers \& Hearn 1989; Pijpers \& Habing 1989), or a combination of pulsation and inefficient radiative cooling of the gas, usually called "pulsationdriven mass loss".

The latter mechanism has been studied by Wood (1979) and Bowen (1988), who showed that shock waves created by the stellar pulsation will successively heat up the gas around the star if the radiative cooling in the outer atmosphere is ineffective. In this case, a hot and slow pressure-driven wind develops, whose mass loss rate depends critically on the assumptions made about the radiative cooling rates (Willson \& Bowen 1998).

Thus, the radiative heating/cooling of the gas is a key quantity to identify the wind driving mechanism of AGB stars. Moreover, since chemistry, nucleation and dust formation depend critically on the temperature structure in the circumstellar environment, the radiative heating/cooling rate as leading source term in the energy equation can also have an important indirect influence on dust-driven winds.

In Woitke et al. (1996a, henceforth Paper I) detailed nonLTE calculations of radiative heating and cooling rates have been presented. These rates have been applied to the problem of shock-induced dust formation around $\mathrm{R}$ Coronae Borealis stars in (Woitke et al. 1996b, henceforth Paper II). In this work, we have implemented an updated and enlarged version of these heating/cooling rates in dynamical model calculations for C-star winds developed by A. Fleischer, J. M. Winters and A. Gauger (the CHILD-Code). These models contain a timedependent description of the dust formation process according to Gail \& Sedlmayr (1988). Thereby, we extend the investigations on circumstellar dust shells around long-period variables carried out in a series of 9 papers starting with Fleischer et al. (1992). We hope that this extension provides some new input for the scientific discussion about the mass loss mechanism of AGB stars.

In Sect. 2, we summarise the system of partial differential equations describing the hydrodynamics, thermodynamics, radiative transfer and dust formation. Section 3 contains a description of the non-LTE state and cooling functions used in this paper. An overview over the numerical method is given in Sect. 4. The results are presented in Sect. 5 and our conclusions are drawn in Sect. 6.

\section{Physical description}

The hydrodynamical model calculations carried out in this paper are based on the CHILD-Code (see introduction). Since the code has been subject to various modifications over the past ten years, we give a brief overview about the current version of the code as used in this paper.

A model is determined by six parameters: $L_{\star}, T_{\star}, M_{\star}$, $\mathrm{C} / \mathrm{O}, P$ and $\Delta u$ denoting the (initial) stellar luminosity, (initial) effective temperature, stellar mass, carbon-to-oxygen ratio, pulsation period and pulsational velocity amplitude. The circumstellar envelope is assumed to be spherically symmetric, thereby reducing the model to one spatial dimension.
A co-moving Lagrangian frame is chosen as coordinate system. The pulsation of the star is simulated by the piston approximation (see Sect. 2.2.1). The circumstellar gas-dust-mixture is regarded as a one-component compressible fluid, i.e. complete position and momentum coupling between gas and dust via frictional forces is assumed. Shock waves are broadened by using the tensor-viscosity of Tscharnuter \& Winkler (1979). The radiative transfer is treated by the time-independent moment method, closed by a two-stream approximation according to (Unno \& Kondo 1976) in the improved version of Hashimoto (1995) for spherical symmetry, grey opacities, local thermodynamical equilibrium (LTE) and radiative equilibrium (for details see Winters et al. 1997). Nucleation, growth and evaporation of dust particles are treated time-dependently, using the moment method developed by Gail \& Sedlmayr (1988) and Gauger et al. (1990) for amorphous carbon dust.

\subsection{Basic equations}

This is the set of equations solved in the current implementation of the code. The meaning of all physical quantities is listed in Appendix B.

- Equation of motion:

$$
\begin{aligned}
& \frac{\mathrm{D} r}{\mathrm{D} t}=u \\
& \frac{\mathrm{D} u}{\mathrm{D} t}=-V_{0}\left(\frac{r}{r_{0}}\right)^{2} \frac{\partial p}{\partial r}-\frac{G M(r)}{r^{2}}(1-\alpha)+\mathcal{F}_{\text {vis }} \\
& \alpha=\frac{\kappa(r) L_{\star}}{4 \pi c G M(r)} ;
\end{aligned}
$$

- Energy equation:

$$
\frac{\mathrm{D} e}{\mathrm{D} t}=-p \frac{\mathrm{D} V}{\mathrm{D} t}+\widehat{Q}_{\mathrm{rad}}+\widehat{Q}_{\mathrm{vis}}
$$

- Radiative transfer:

$$
\begin{aligned}
T_{\text {rad }}{ }^{4}= & \frac{r^{2} F\left(r_{\text {inner }}\right)}{2 \sigma} \cdot\left[\frac{1}{R^{2}\left(1+\mu_{R}\right)}+\frac{\mu_{R}}{R^{2}}-\frac{\mu_{r}}{r^{2}}\right. \\
& \left.+\frac{3}{2} \int_{r}^{R}\left(\kappa\left(r^{\prime}\right)+\frac{2 \mu_{r^{\prime}}}{r^{\prime}}\right) \frac{\mathrm{d} r^{\prime}}{r^{\prime 2}}\right] \\
\frac{\mathrm{d} \mu_{r}^{3}}{\mathrm{~d} r}= & \frac{\kappa(r)}{4}\left(1-5 \mu_{r}^{2}\right)+\frac{3 \mu_{r}}{r}\left(1-\mu_{r}^{2}\right) ;
\end{aligned}
$$

- Dust moment equations:

$$
\begin{aligned}
\frac{\mathrm{D} K_{0}}{\mathrm{D} t} & =\frac{J\left(N_{\mathrm{l}}\right)}{n_{\langle\mathrm{H}\rangle}} \\
\frac{\mathrm{D} K_{\mathrm{j}}}{\mathrm{D} t} & =N_{\mathrm{l}}^{j / 3} \frac{J\left(N_{\mathrm{l}}\right)}{n_{\langle\mathrm{H}\rangle}}+\frac{j}{3 \tau_{\text {net }}} K_{\mathrm{j}-1} .
\end{aligned}
$$

Note that in the Lagrangian formulation, the continuity equation is automatically fulfilled according to Eq. (1). 


\subsection{Boundary and initial conditions}

\subsubsection{Hydrodynamical initial and boundary conditions}

Inner boundary: The inner boundary is situated a few scale heights below the initial stellar radius. In order to simulate the pulsation of the star, a sinusoidal variation of the velocity at the inner boundary is assumed (piston approximation, Wood 1979)

$u\left(r_{\text {inner }}\right)=\Delta u \cos \left(\frac{2 \pi t}{P}\right)$.

Outer boundary: A constant (pressure-) gradient condition is applied in order to determine the velocity at the outermost grid point (see Fleischer et al. 1992, p. 324).

Initial condition: The dynamical calculations are started from a dust-free solution of the basic Eqs. (2)-(6) in the hydrostatic case and radiative equilibrium, which is obtained on the same grid with the same discretisation scheme (in the following referred to as the start model).

\subsubsection{Boundary and consistency conditions for the radiative transfer}

Inner boundary: The frequency-integrated radiative flux at the inner boundary $F\left(r_{\text {inner }}\right)$ is assumed to be constant, thereby fixing the stellar luminosity as function of time according to the position of the inner boundary $L_{\star}(t)=4 \pi r_{\text {inner }}^{2}(t) F\left(r_{\text {inner }}\right)$. The separation angle at the inner boundary is set zero

$\mu\left(r=r_{\text {inner }}\right)=0$.

Outer boundary: The optical depth is forced to vanish at the outer boundary. Outer irradiation is neglected.

Consistency condition: The position of the stellar radius $R_{\star}(t)$ and the effective temperature $T_{\star}(t)$ are calculated from the Stefan-Boltzmann-law and a consistency condition at $r=R \star$

$L_{\star}=4 \pi R_{\star}^{2} \sigma T_{\star}^{4}, \quad T_{\mathrm{rad}}\left(r=R_{\star}\right)=T_{\star}$

which states two equations for the two unknowns $R_{\star}(t)$ and $T_{\star}(t)$. These quantities, however, have only informal character and are not used elsewhere. $T_{\mathrm{rad}}(r)$ is the grey radiative equilibrium temperature structure calculated according to Eq. (5).

\subsection{Material functions}

The calculation of the source terms emerging in the basic Eqs. (2) to (8) requires the knowledge of several material functions. These material functions, which describe the couplings between hydrodynamics, thermodynamics, radiative transfer, chemistry and dust formation, are determined from various microphysical considerations. In the following, we list the required material functions along with a short description of the underlying concepts for their determination.

- The thermal and the caloric equations of state describe the dependence of the gas pressure $p$ and the internal energy $e$ on the fundamental thermodynamical state variables

$$
\begin{aligned}
p & =p\left(T_{\mathrm{g}}, \rho, \ldots\right) \\
e & =e\left(T_{\mathrm{g}}, \rho, \ldots\right) .
\end{aligned}
$$

The state functions used in this work emerge from the time-independent non-LTE description of the gas outlined in Sect. 3.1.

- The net radiative heating rate $\widehat{Q}_{\text {rad }}$ describes the energy exchange between the gas and the radiation field. According to the concept outlined in Paper I, $\widehat{Q}_{\text {rad }}$ is the net flux of photon energy from the radiation field to the gas (the sum of black arrows in Fig. 1 of Paper I)

$\widehat{Q}_{\mathrm{rad}}=\widehat{Q}_{\mathrm{rad}}\left(T_{\mathrm{g}}, \rho, \ldots\right)$.

The value of $\widehat{Q}_{\text {rad }}$ is determined together with the other state functions as described in Sect. 3.1. The influence of $\widehat{Q}_{\text {rad }}$ on the resulting model structures is the primary focus of this paper.

- The net flux of dust particles in size-space at the lower integration boundary $N_{1}$

$J\left(N_{1}\right)=J_{\star}-J_{\text {evap }}$

accounts for the crossing of dust particles through the lower integration boundary of the dust moments $K_{j}$. In case of net growth, $J\left(N_{1}\right)$ is identified with the nucleation rate $J_{\star}$ as calculated by modified classical nucleation theory (Gail et al. 1984). In case of net evaporation, $J_{\text {evap }}$ is calculated from the cluster size distribution function at $N_{1}$ as reconstructed from the history of $J_{\star}$ and $\tau_{\text {net }}$ in the considered mass element (for details see Gauger et al. 1990).

- The net growth rate

$\frac{1}{\tau_{\text {net }}}=\frac{1}{\tau_{\text {gr }}}-\frac{1}{\tau_{\text {evap }}}$

describes the growth and evaporation of dust particles (see Gauger et al. 1990). The calculation of $J\left(N_{1}\right)$ and $\tau_{\text {net }}$ requires the knowledge of certain molecular particle densities $\left(\mathrm{C}, \mathrm{C}_{2}, \mathrm{C}_{2} \mathrm{H}, \mathrm{C}_{2} \mathrm{H}_{2}\right)$ involved in the nucleation and surface chemical processes. These concentrations are determined by assuming chemical equilibrium in the gas phase according to the local element abundances and assuming that oxygen is completely blocked by $\mathrm{CO}$. The element abundance of carbon is reduced according to the consumption by dust formation $\epsilon_{\mathrm{C}}=\epsilon_{\mathrm{C}}(t=0)-K_{3}$.

- The total extinction coefficient

$\kappa=\kappa_{\text {dust }}+\kappa_{\text {gas }}$

describes the absorption and scattering of photons by the gas-dust-mixture. In this work we use the constant gas opacity proposed by Bowen (1988)

$\kappa_{\text {gas }}=\rho \cdot 2 \times 10^{-4} \mathrm{~cm}^{2} \mathrm{~g}^{-1}$.

We follow this approach, in order to focus on the effects introduced by the new state functions. This facilitates a comparison to the results of earlier works (e.g. Fleischer et al. 1992; Winters et al. 1997; Höfner et al. 1995), who have adopted the same value. The influence of a variable gas opacity on the structures of dynamical CSEs of AGB stars has been investigated by Helling et al. (2000), in the 
framework of a grey radiative transport, and by Höfner et al. (2003), who have recently presented models with a frequency-dependent radiative transfer. The total dust opacity is calculated in the small particle limit of Mie theory:

$\kappa_{\text {dust }}=n_{\langle\mathrm{H}\rangle} \cdot \frac{3}{4} V_{0} K_{3} Q^{\prime}\left(T_{\text {dust }}\right)$,

$V_{0}$ is the dust volume per monomer and $Q^{\prime}\left(T_{\text {dust }}\right)=$ $4.4 T_{\text {dust }}$ the Rosseland mean of the extinction efficiency of the dust grain material divided by the grain radius $a$ in the small particle limit (see Gail \& Sedlmayr 1987b; Helling 1999). The dust temperature is assumed to equal the grey radiative equilibrium temperature $T_{\text {dust }}=T_{\text {rad }}$.

\section{The state functions}

\subsection{Time-independent non-LTE description}

In the following, we resume the basic concept of non-LTE state functions (see also Mihalas 1978, p. 140) for partly molecular gases under the influence of a continuous radiation field as developed in Paper I and describe the updates and improvements made since Paper I.

In comparison to the standard LTE approach, where $\rho$ and $T_{\mathrm{g}}$ are sufficient to determine the complete state of the gas, including all relevant particle densities and occupational numbers, we consider the mean spectral intensity $J_{v}$ as (a set of) $a d$ ditional fundamental thermodynamical variable(s). From these variables, all gain and loss terms in the non-LTE statistical equations can be determined, such that the time-independent solution of these equations can be determined. By relying on the time-independent case, we assume that the internal relaxation by atomic and molecular processes (e.g. dissociation, excitation and ionisation) is fast in comparison to changes of the thermodynamical variables in the ambient medium (e.g. due to velocity fields). The non-LTE statistical equations are completed by the requirement of total particle and charge conservation, describing the chemical conversions between different kinds of particles.

A high-dimensional system of algebraic equations is obtained, which is solved by iteration. The result is a vector of molecular, atomic and ionic particle densities, including all occupational numbers, and the electron density, from which all relevant state functions can be computed. This approach allows for an inclusion of various important non-LTE effects, like photo-ionisation and the depopulation of excited states in the case of small densities and weak radiation fields. Timedependent non-LTE effects, however, cannot be described by this approach.

For this work, the outlined concept for the calculation of the state of the gas is carried out with the following additional approximations:

1. For the sake of simplicity, we assume chemical equilibrium between molecules and neutral atoms. Our approach could, in principle, also be carried out by calculating the time-independent solution of a chemical rate network, but this procedure would be very time-consuming and goes beyond our current capabilities ${ }^{1}$. In contrast, the ratios between ions and neutral atoms are properly calculated in non-LTE.

2. Optical depth effects in spectral lines are treated by using an escape probability method according to Sobolev theory (e.g. Puls \& Hummer 1988). In doing so, we can replace the usually unknown local mean intensities $J_{v}$ by other quantities again regarded as fundamental thermodynamical variables (see Eq. (12) in Paper I), namely (i) the local continuous mean intensity $J_{v}^{\text {cont }}$, and (ii) the local mean velocity gradient $\left\langle\frac{\mathrm{d} v}{\mathrm{~d} l}\right\rangle$. Both are available in radiationhydrodynamical simulations.

3. Our hydrodynamical models only include a grey continuum radiative transfer, where detailed knowledge about the frequency distribution of $J_{v}^{\text {cont }}$ is not available, but only its frequency integral $J^{\text {cont }}=\sigma T_{\text {rad }}^{4} / \pi$ is known. In order to proceed further, we assume

$$
J_{v}^{\text {cont }}=B_{v}\left(T_{\text {rad }}\right)
$$

which is strictly correct only in the optically thick limiting case $^{2}$. The set of variables for $J_{v}^{\text {cont }}$ is hence simplified to a single variable, namely $T_{\text {rad }}$, and the complete set of fundamental thermodynamical variables needed to calculate the state of the gas is reduced to $\rho, T_{\mathrm{g}}, T_{\text {rad }}$ and $\left\langle\frac{\mathrm{d} v}{\mathrm{~d} l}\right\rangle$.

With these assumptions, all particle densities (neutral atoms, molecules, electrons and single ions) and level populations are determined in statistical equilibrium. The resulting state functions are calculated and tabulated as function of our four fundamental thermodynamical variables. This concerns the internal energy of the gas $e$, the gas pressure $p$ and the net radiative heating function $\widehat{Q}_{\text {rad }}$. These three four-dimensional tables are used later in the hydrodynamical model calculations.

An overview of the included photo-processes for the calculation of $\widehat{Q}_{\text {rad }}$ is given in Table 1 . The resulting magnitude and importance of the various radiative processes in the temperature-density-plane can be found in Woitke \& Schirrmacher (1999).

Concerning the non-LTE treatment of the neutral atoms and first ions, we use the same methods and equations as presented in Paper I, but the atomic data has been updated and considerably enlarged as described in (Woitke \& Sedlmayr 1999). The model atoms now include several thousand permitted, forbidden and fine-structure lines, and $b$ - $f$ transitions from excited levels. New species like Ca I, Ca II, Mg I and Mg II are taken

\footnotetext{
${ }^{1}$ We note that chemical non-equilibrium effects in the timeindependent case may even lead to spurious effects since existing chemical rate networks do usually not lead to the chemical equilibrium solution for $t \rightarrow \infty$, even if $J_{v}=B_{v}\left(T_{\mathrm{g}}\right)$ is assumed, which contradicts very basic thermodynamical considerations. The reason is that usually the corresponding forward and reverse processes are either not included pair-wise in all cases or that the respective rate coefficients are not properly related to each other via the Gibb's free energies of the molecules, but rather treated in an independent way.

${ }^{2}$ Other, eventually multi-parametric descriptions of $J_{v}^{\text {cont }}$ are also conceivable, e.g. adjusted to the optically thin case.
} 
Table 1. Overview of included heating/cooling processes.

\begin{tabular}{|c|c|c|}
\hline Carrier & Type of transitions & Ref. \\
\hline $\begin{array}{l}\text { Neutral atoms and } \\
\text { first ions (13 ele- } \\
\text { ments) }\end{array}$ & bound-free and line transitions & 1 \\
\hline Positive ions & free-free (bremsstrahlung) & 2 \\
\hline $\mathrm{H}^{-}$ & bound-free and free-free & $3,4,5$ \\
\hline $\mathrm{H}_{2}^{-}, \mathrm{He}^{-}, \mathrm{C}^{-}$ & free-free & $6,7,8$ \\
\hline $\begin{array}{l}\mathrm{H}_{2} \mathrm{O}, \quad \mathrm{CO}_{2}, \quad \mathrm{HCN}, \\
\mathrm{C}_{2} \mathrm{H}_{2}, \quad \mathrm{H}_{2} \mathrm{~S}, \quad \mathrm{SO}_{2}, \\
\text { OCS }\end{array}$ & $\begin{array}{l}\text { rotational, ro-vibrational } \\
\text { including overtones and combi- } \\
\text { nation bands }\end{array}$ & App. A \\
\hline $\mathrm{CO}, \mathrm{SiO}, \mathrm{CS}$ & 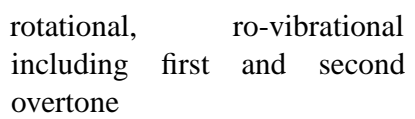 & App. A \\
\hline $\begin{array}{l}\mathrm{CN}, \mathrm{OH}, \mathrm{CH}, \mathrm{SO} \\
\mathrm{HS}, \mathrm{SiS}, \mathrm{SiN}, \mathrm{SiC}\end{array}$ & $\begin{array}{l}\text { rotational and fundamental } \\
\text { mode ro-vibrational }\end{array}$ & 5 \\
\hline $\mathrm{C}_{2} \mathrm{H}, \mathrm{C}_{2} \mathrm{~N}$ & rotational & 5 \\
\hline $\mathrm{H}_{2}, \mathrm{~N}_{2}$ & ro-vibrational (quadrupole) & App. A \\
\hline
\end{tabular}

(1) Woitke \& Sedlmayr (1999) and references therein; (2) Hummer (1988); (3) Wishart (1979); (4) Stilley \& Callaway (1970); (5) Paper I and references therein; (6) Somerville (1964); (7) John (1994); (8) John \& Williams (1977).

into account (see Table 1 in Woitke \& Sedlmayr 1999). In addition to the photo-ionisation and direct recombination rates, collisional ionisation from all neutral (ground and excited) levels is included, affecting e.g. the determination of the electron particle densities which is important for the collision rates.

Concerning the treatment of the molecules, several updates and methodological improvements have been carried out since Paper I, which are outlined in Appendix A. Polyatomic molecules like $\mathrm{H}_{2} \mathrm{O}, \mathrm{C}_{2} \mathrm{H}_{2}, \mathrm{HCN}$ and $\mathrm{CO}_{2}$ are taken into account and the non-LTE treatment has been refined in order to allow for the inclusion of overtone transitions, combination bands, and the important ro-vibrational pumping effect.

\subsection{Resulting non-LTE state functions}

Thermal equation of state: Figure 1 shows the resulting dependence of the thermal gas pressure $p$ as calculated by

$p=\left(n_{\text {atom }}+n_{\text {mol }}+n_{\text {ion }}+n_{\mathrm{el}}\right) k_{\mathrm{B}} T_{\mathrm{g}}$

$$
\text { with } n_{\mathrm{i}}=n_{\mathrm{i}}\left(T_{\mathrm{g}}, \rho, T_{\mathrm{rad}},\left\langle\frac{\mathrm{d} v}{\mathrm{~d} l}\right\rangle\right)
$$

on the gas temperature $T_{\mathrm{g}}$ for a collection of different gas densities $\rho$. The lower panel of Fig. 1 shows the resulting mean molecular weight $\mu$ calculated by

$\mu=\frac{\rho k_{\mathrm{B}} T_{\mathrm{g}}}{p\left(T_{\mathrm{g}}, \rho, T_{\mathrm{rad}},\left\langle\frac{\mathrm{d} v}{\mathrm{~d} l}\right\rangle\right)}$.

At low temperatures $\mu$ is dominated by the ratio of $\mathrm{H}_{2}$ to $\mathrm{H}$ atoms ${ }^{3}$, at high temperatures by ionisation of abundant

\footnotetext{
${ }^{3}$ In this paper, we rely on the mean molecular weight $\mu$ as indicator for the dissociative state of hydrogen, because in our dynamical calculations, the state functions enter in a tabulated form where the actual particle densities $n_{\mathrm{i}}$ are "forgotten".
}

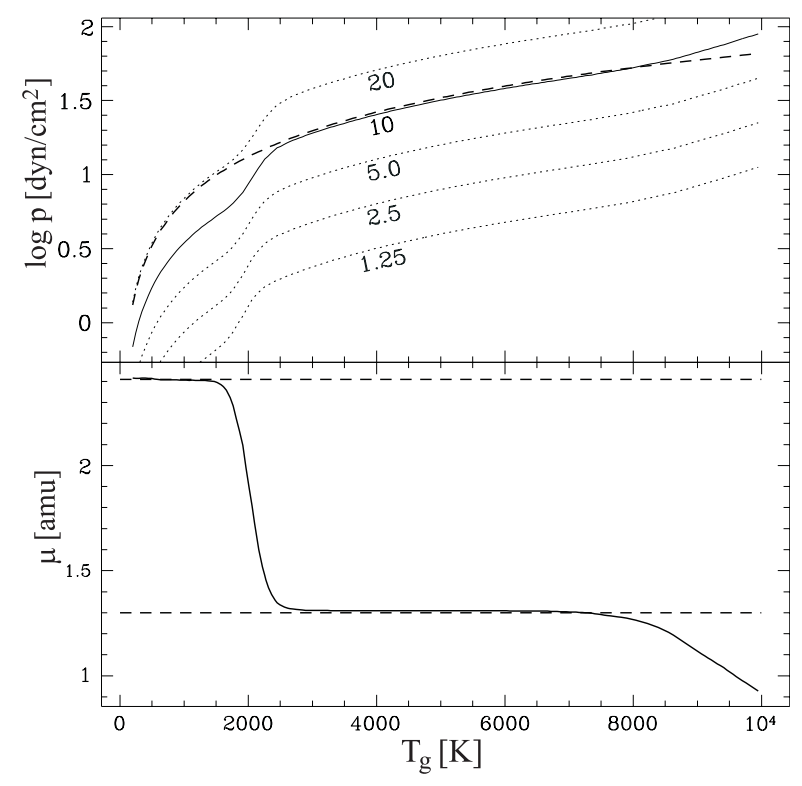

Fig. 1. Upper panel: Dependence of the thermal gas pressure $p$ on the gas temperature $T_{\mathrm{g}}$ for various densities in units of $10^{-11} \mathrm{~g} \mathrm{~cm}^{-3}$ (solid and dotted lines as indicated). $T_{\mathrm{rad}}=1500 \mathrm{~K}$ and $\left\langle\frac{\mathrm{d} v}{\mathrm{~d} l}\right\rangle=10^{-7} \mathrm{~s}^{-1}$ are fixed parameters. For comparison, the dashed line shows an ideal mono-atomic gas $\left(\mu=1.3 m_{\mathrm{H}}\right)$ for $\rho=10^{-10} \mathrm{~g} \mathrm{~cm}^{-3}$. Lower panel: Mean molecular weight $\mu$ in atomar mass units over gas temperature for $\rho=10^{-10} \mathrm{~g} \mathrm{~cm}^{-3}$. The upper dashed line corresponds to the value of $\mu$ where all hydrogen is bound in $\mathrm{H}_{2}$, the lower dashed line to atomic hydrogen.

metal atoms and, in particular, of hydrogen. Clearly, an ideal gas with constant $\mu$ (sometimes assumed in other models) is not appropriate for the modelling of AGB star winds, as it would introduce an error of a factor $\sim 2$.

Caloric equation of state: The total internal energy is calculated by summing up the various contributions from all internal pools of energy, including potential energies due to ionisation and dissociation

$$
\begin{gathered}
e=e_{\text {trans }}+e_{\mathrm{rot}}+e_{\mathrm{vib}}+e_{\mathrm{el}}+e_{\mathrm{diss}}+e_{\mathrm{ion}} \\
\text { with } e_{\mathrm{i}}=e_{\mathrm{i}}\left(T_{\mathrm{g}}, \rho, T_{\mathrm{rad}},\left\langle\frac{\mathrm{d} v}{\mathrm{~d} l}\right\rangle\right) .
\end{gathered}
$$

The different forms of internal energy $e_{i}$ are calculated according to Eqs. (3) to (8) in Paper I. The dependence of the internal energy on the gas temperature $T_{\mathrm{g}}$ is shown in Fig. 2. Again, the assumption of an ideal mono-atomic gas yields realistic values only in those domains where hydrogen is actually mainly atomic. Two distinct steps are caused by the dissociation and the ionisation of hydrogen. The slope of $e\left(T_{\mathrm{g}}\right)$ is the specific heat capacity $c_{\mathrm{V}}$ of the gas, defined by

$$
c_{\mathrm{V}}=\left.\frac{\partial e\left(T_{\mathrm{g}}, \rho, T_{\mathrm{rad}},\left\langle\frac{\mathrm{d} v}{\mathrm{~d} l}\right\rangle\right)}{\partial T_{\mathrm{g}}}\right|_{T_{\mathrm{rad}}, \rho,\left\langle\frac{\mathrm{d} v}{\mathrm{~d}}\right\rangle=\mathrm{const}},
$$

which peaks in the phase transition regions between atomic and molecular hydrogen, and between atomic and ionised hydrogen. 


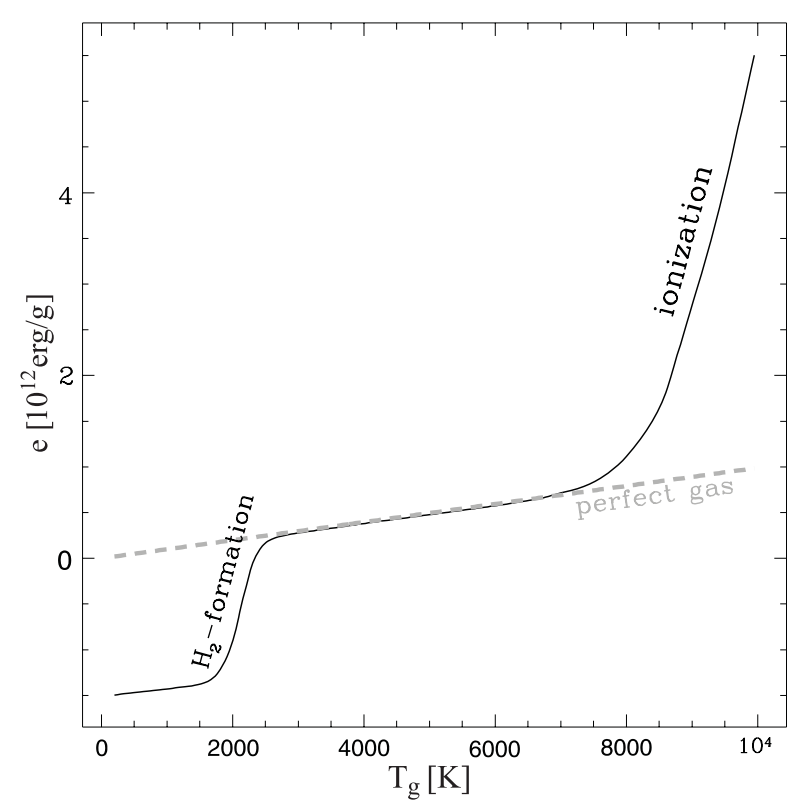

Fig. 2. Internal energy $e$ over gas temperature $T_{\mathrm{g}}$ (solid line) for constant $\rho=10^{-10} \mathrm{~g} \mathrm{~cm}^{-3}, T_{\text {rad }}=1500 \mathrm{~K}$ and $\left\langle\frac{\mathrm{d} v}{\mathrm{~d} l}\right\rangle=10^{-7} \mathrm{~s}^{-1}$. For comparison, the internal energy of an ideal mono-atomic gas is shown as dashed line.

Both equations of state reveal essentially the same behaviour as used from LTE calculations. However, the dependencies are considerably affected by the ionisation equilibrium (mainly triggered the balance between photo-ionisation and direct recombination) and, thus, also depend on the radiation field (not shown in Figs. 1 and 2).

Net radiative heating rate: $\widehat{Q}_{\text {rad }}$ varies by orders of magnitude, changes its sign and depends strongly on all four parameters $T_{\mathrm{g}}, \rho, T_{\mathrm{rad}}$ and $\left\langle\frac{\mathrm{d} v}{\mathrm{~d} l}\right\rangle$. Figures 3 and 4 give a rough impression of the dependencies on $T_{\mathrm{g}}$ and $\rho$ in the case $T_{\mathrm{rad}}=0$, i.e. with negligible continuous radiation field where, clearly, the gas cools by radiative losses. Other illustrative examples are shown in Woitke \& Sedlmayr (1999) and in Woitke \& Schirrmacher (1999).

Figure 3 shows that $\widehat{Q}_{\text {rad }}$ usually has a maximum around $\approx 10^{-13} \mathrm{~g} \mathrm{~cm}^{-3}$. Towards lower densities, the radiative cooling becomes less effective because of the decreasing collisional excitation rates. Towards higher densities, the optical depths in the spectral lines generally increase which results in a significant trapping of the emitted line photons.

Figure 4 demonstrates that $\widehat{Q}_{\text {rad }}$ does usually not follow a simple relaxation ansatz like $\widehat{Q}_{\mathrm{rad}} \propto T_{\mathrm{rad}}-T_{\mathrm{g}}$ or $\widehat{Q}_{\mathrm{rad}} \propto T_{\mathrm{rad}}^{4}-T_{\mathrm{g}}^{4}$. On the contrary, the resulting cooling rates generally have a local maximum around $2000 \mathrm{~K}$... $3000 \mathrm{~K}$ where molecules are present in the gas phase, which are effective coolants because of their numerous ro-vibrational transitions, in particular the polar and abundant $\mathrm{CO}$-molecule: The radiative cooling rate can re-increase with decreasing gas temperature.

\section{The numerical method}

A detailed description of the CHILD-Code can be found in Fleischer et al. (1992) and Winters et al. (1997). We therefore give only a short summary here.

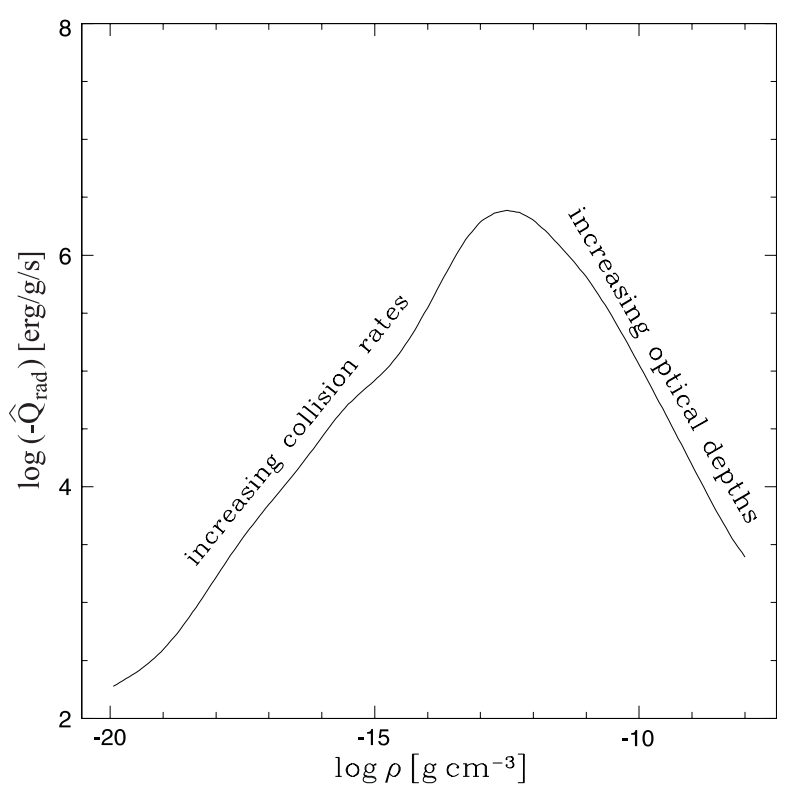

Fig. 3. Radiative cooling rate $-\widehat{Q}_{\text {rad }}$ over gas density $\rho$ for $T_{\text {rad }}=0$, $T_{\mathrm{g}}=4000 \mathrm{~K}$ and $\left\langle\frac{\mathrm{d} v}{\mathrm{~d} l}\right\rangle=10^{-7} \mathrm{~s}^{-1}$.

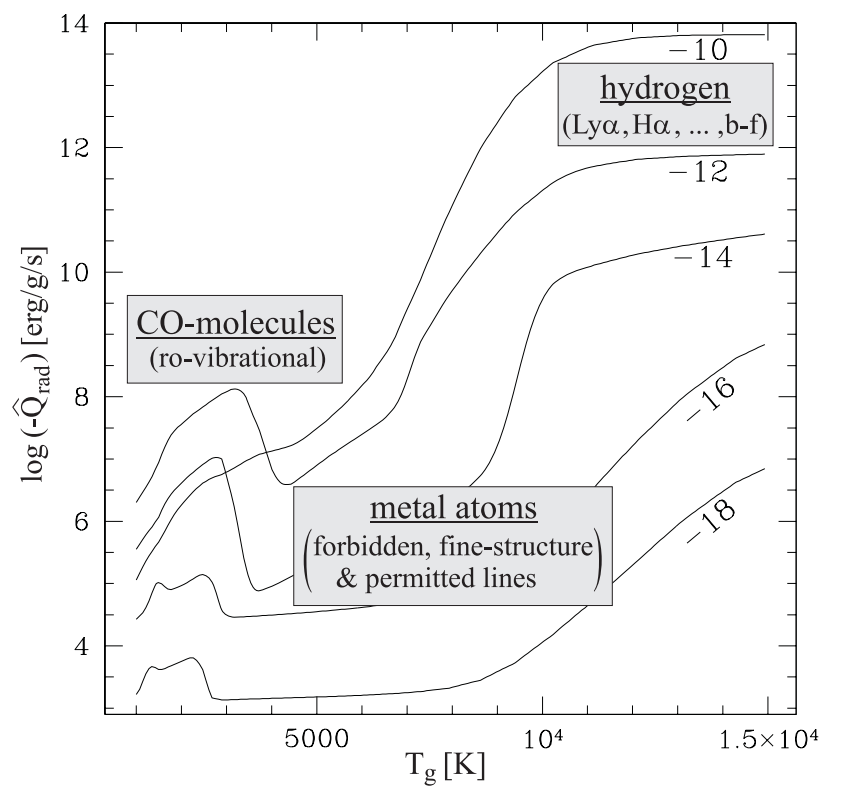

Fig. 4. Radiative cooling rate $-\widehat{Q}_{\text {rad }}$ over gas temperature $T_{\mathrm{g}}$ for $T_{\text {rad }}=$ $0,\left\langle\frac{\mathrm{d} v}{\mathrm{~d} l}\right\rangle=10^{-7} \mathrm{~s}^{-1}$ and different gas densities ( $\log \rho$ as indicated). Note that this case is only one example. An increase of the continuous radiation field will, for example, result in more photoionisation and hence to an increase of the electron concentration which usually intensifies the radiative cooling.

The equation system described in Sect. 2.1 is discretised on a staggered mesh according to the scheme of Richtmyer \& Morton (1967), and solved by explicit forward integration in time. The length of the time-steps is adapted dynamically, in order to handle the rapid evolution of the dust component in phases when new dust shells condense. The fraction of the maximum allowed CFL-time step is set to $5 \times 10^{-2}$. A stellar period is typically calculated in $10^{5}$ time steps. A rezoning mechanism is implemented (see Fleischer et al. 1992), which 
discards zones that cross an arbitrary outer limit and insert new grid points by splitting zones ${ }^{4}$ in regions where the gradients are steep.

The dynamical calculations are started by switching on the motion of the piston at the inner boundary. The velocity amplitude is carefully incremented until the desired value is reached, in order to reduce the effects caused by the very first shock waves which steepen up dramatically in the initially hydrostatic atmosphere. The number of grid points, initially 512, is gradually increased to 1925 by inserting more and more points via the rezoning procedure. For the models presented in this paper, we have fixed the outer boundary at $25 R_{0}$, thus assuring a satisfactory resolution behind the shock waves. The results are taken after the pulsation amplitude and the number of grid points have reached their final value, and after several further shock waves, generated close to the star, have reached the outer boundary.

The new state functions are interpolated from 4dimensional tables for $\ln \left(\frac{p}{\rho T_{\mathrm{g}}}\right), \ln \left(\frac{\widehat{Q}_{\text {rad }}}{T_{\mathrm{g}}-T_{\text {rad }}}\right)$ and $e$. The variables are $T_{\mathrm{g}}, T_{\mathrm{rad}}, \rho$ and $\left\langle\frac{\mathrm{d} v}{\mathrm{~d} l}\right\rangle$ on a $50 \times 30 \times 48 \times 6$-grid. $T_{\mathrm{g}}, \rho$ and $\left\langle\frac{\mathrm{d} v}{\mathrm{~d} l}\right\rangle$ run on a logarithmic spacing $\left(300 \mathrm{~K}<T_{\mathrm{g}}<20000 \mathrm{~K}\right.$, $10^{-20} \mathrm{~g} \mathrm{~cm}^{-3}<\rho<10^{-8} \mathrm{~g} \mathrm{~cm}^{-3}$ and $\left.10^{-10} \mathrm{~s}^{-1}<\left\langle\frac{\mathrm{d} v}{\mathrm{~d} l}\right\rangle<10^{-6} \mathrm{~s}^{-1}\right)$, while for $T_{\text {rad }}$ we chose a spacing that ensures a high density of points around $T_{\text {rad }}=1500 \mathrm{~K}\left(0 \mathrm{~K}<T_{\text {rad }}<5000 \mathrm{~K}\right)$.

The main loop of the forward integration consists of the following steps:

1. Determination of the time step $\Delta t$. For details of the adapted criteria see Fleischer (1994).

2. Calculation of the new hydrodynamic structure $r_{\text {new }}$, $u_{\text {new }}, \rho_{\text {new }}$ and $\left\langle\frac{\mathrm{d} v}{\mathrm{~d} l}\right\rangle_{\text {new }}$ according to Eqs. (1), (2), mass conservation and Eq. (17) of Paper I.

3. Determination of the new gas temperatures $T_{\mathrm{g}, \text { new }}$ by means of the semi-implicitly discretised energy Eq. (4). In the internal iteration required to find the root of this equation, the quantities $e_{\text {new }}, p_{\text {new }}$ and $\widehat{Q}_{\text {rad,new }}$ are interpolated from the 4-dimensional tables as functions of $\rho_{\text {new }}, T_{\mathrm{g}, \text { new }}$, $\left\langle\frac{\mathrm{d} v}{\mathrm{~d} l}\right\rangle_{\text {new }}$ and $T_{\text {rad,old }}$.

4. Determination of the new grey radiative equilibrium temperature structure $T_{\text {rad,new }}$ by means of the solution of Eqs. (5) and (6), where the dust opacities are iteratively determined according to $T_{\text {rad,new }}$ (Winters et al. 1997).

5. Calculation of the dust complex. The new chemical concentrations of the dust forming species are calculated according to $\rho_{\text {new }}$ and $T_{\text {g,new }}$ and the dust moment Eqs. (7) and (8) are explicitly integrated forward.

6. Validation of the time step. The artificial viscosities and radiative acceleration $\alpha_{\text {new }}$ for the next time step are evaluated, taking into account the new opacities which depend on the new dust moments and the new temperatures. The time step is discarded if the resulting change of any quantity is too large.

4 Actually no single zones are split, but three new zones are carefully constructed out of two old ones, thus preventing numerical generation of mass or momentum.

\section{Results and discussion}

\subsection{Hydrostatic start models}

Before we present the results of our dynamical models which include a non-LTE treatment of the state functions as described in Sect. 3, we start with a short discussion of the start models. These hydrostatic structures already reveal some remarkable deviations from the former calculations, where isothermal or LTE radiative cooling was assumed and the state functions of a perfect mono-atomic gas were considered (henceforth called the classical models, see e.g. Fleischer et al. 1992; Winters et al. 2000).

Figure 5 depicts the calculated hydrostatic density stratification in comparison with two start models calculated with a constant mean molecular weight, corresponding to a $\mathrm{H} / \mathrm{He}$ mixture ("mono-atomic") and $\mathrm{a}_{2} / \mathrm{He}$ mixture ("di-atomic"), respectively. The plot demonstrates that the new density structure lies between these two limiting cases and differs from the classical start model (which considers a perfect mono-atomic gas) by about two orders of magnitude in the outer regions.

The reason for the lower densities in the outer regions of the new models is the changing $\mathrm{H} / \mathrm{H}_{2}$ ratio as indicated by the lower plot of Fig. 5. As the temperature decreases outward in the inner atmospheric layers, molecular hydrogen starts to form. However, although the temperature still decreases slowly in the outer regions, the pressure gradient in the hydrostatic case is so steep, that the dissociative equilibrium of $\mathrm{H}_{2}$ finally favours atomic hydrogen again. In the region around the stellar radius, where $\mu$ has a pronounced maximum, the logarithmic density gradient is about a factor 2 steeper since 5

$H_{\mathrm{p}}^{-1}=\left|\frac{\partial \ln p}{\partial r}\right|=\frac{\mu g}{k T}$.

Consequently, the density starts to fall behind the classical mono-atomic model outside of about $1 R_{\star}$.

Thus, the new models already show distinct deviations from the classical models in the hydrostatic case. A detailed comparison of single pairs of classical and new models for the same parameters, e.g. at a certain instant of time, is hence a lot less meaningful than suggested by intuition. Therefore, after reviewing the results of the new model calculations in general (Sect. 5.2), we will mainly focus on the new physical effects introduced by the refined state and cooling functions ${ }^{6}$.

\subsection{Dynamical models - An overview}

The results of the new dynamical model calculations generally reveal a dust-driven wind triggered by the pulsation of the star, similar to the classical models. Sound waves initiated by the sinusoidal variation of the inner boundary quickly steepen up to strong shock waves in the large density gradient of the photosphere. For the relatively compact AGB star with Mira-like

\footnotetext{
5 Neglecting the difference between $H_{\mathrm{p}}$ and $H_{\rho}$.

6 A detailed statistical comparison between new and classical models was not the aim of this work, but might be presented in a subsequent paper.
} 

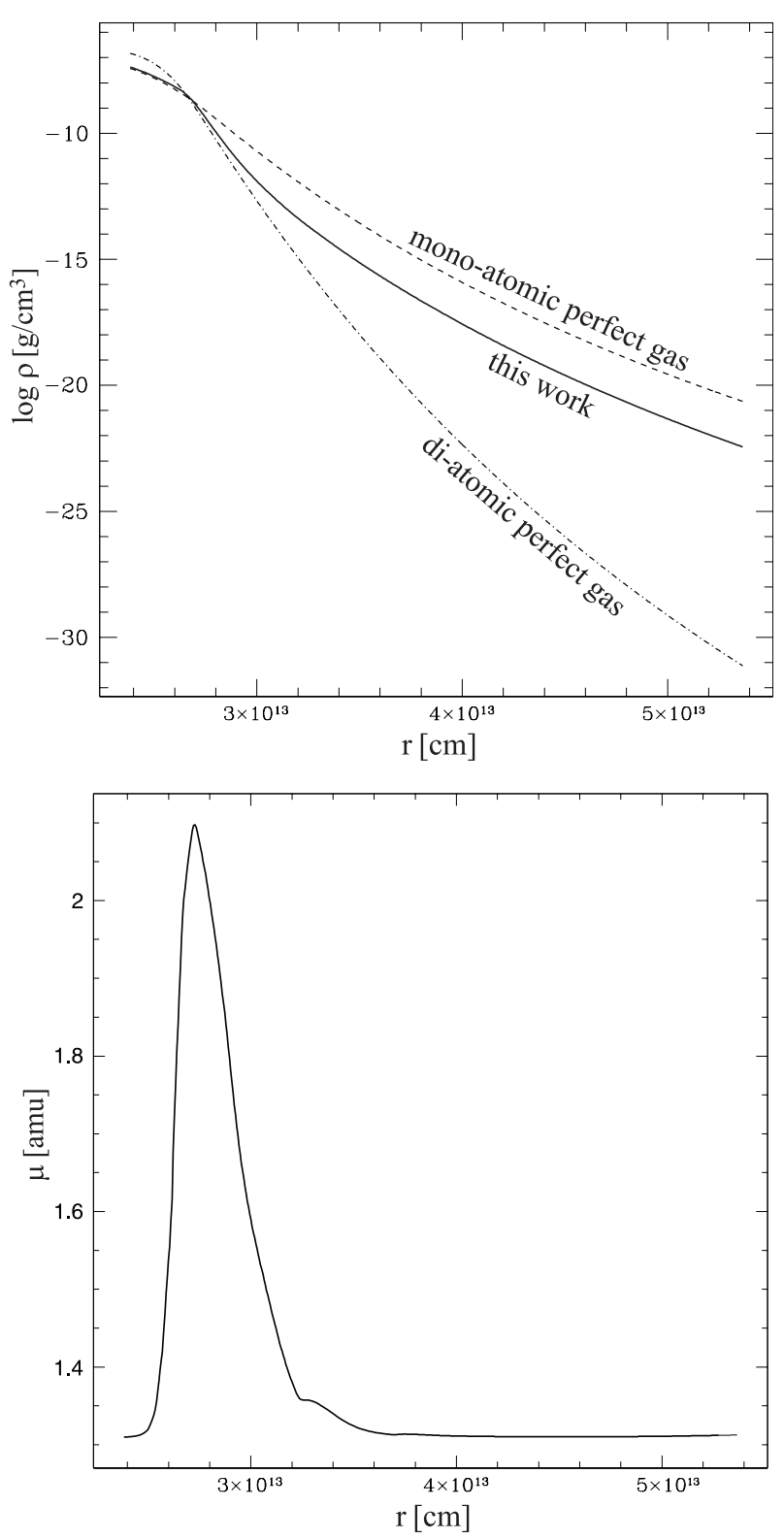

Fig. 5. Start models for parameters $M_{\star}=1.2 M_{\odot}, L_{\star}=6125 L_{\odot}$ and $T_{\star}=2600 \mathrm{~K}$ (derived quantities $R_{\star}=2.68 \times 10^{13} \mathrm{~cm}, \log g=$ -0.66) compared with two start models calculated with constant $\mu=$ $1.26 \mathrm{amu}\left(\mathrm{H} / \mathrm{He}\right.$ mixture, dashed) and $\mu=2.33 \mathrm{amu}\left(\mathrm{H}_{2} / \mathrm{He}\right.$ mixture, dashed-dotted).

parameters ${ }^{7}$ considered in Fig. $6^{8}$, shock waves with velocities up to $35 \mathrm{~km} \mathrm{~s}^{-1}$ develop slightly above the time-dependent position of the stellar radius $R_{\star}(t)$ (see Eq. (11)). While propagating outwards through the dust-free regions close to the star, the shock waves are damped and strongly levitate the outer atmosphere. When a shock wave reaches the dust formation zone around $2 \ldots 3 R_{0}$, this zone is compressed which temporarily creates favourable conditions for dust formation. Radiation pressure on the newly formed dust grains then pushes the gasdust-mixture outwards, thus providing the basic wind driving

\footnotetext{
7 The choice of these parameters was guided by investigations of (Richter et al. 2002) on Fe II emission lines.

${ }^{8}$ See also http://astro.physik.tu-berlin.de/ woitke/ Mira.mpeg.
}

mechanism in these models. The dynamic coupling between pulsation and time-dependent dust formation usually leads to the production of radial dust shells in sometimes periodic, but often multi-periodic or chaotic time intervals (see lower box of Fig. 6). The dust-forming shock waves are re-accelerated which causes density inversions to develop in the post-shock regions.

In contrast to the classical models, several new features appear in the model calculations which are further discussed in the Sects. 5.3 to 5.5. The most striking difference is the occurrence of high temperature peaks around the positions of the strongest shock waves. If the pre-shock gas is $\mathrm{H}_{2}$-rich, only shock waves $\gtrsim 20 \mathrm{~km} \mathrm{~s}^{-1}$ are energetically capable to completely dissociate the $\mathrm{H}_{2}$ molecules. The surplus energy dissipated by the shock is then converted into thermal kinetic energy, leading easily to post-shock temperatures of about $8000 \mathrm{~K}$ to $11000 \mathrm{~K}$. Shock waves below this threshold (non-dissociative shocks) usually produce much smaller postshock temperatures.

The double box in the centre of Fig. 6 gives an overview of the energetic processes of the gas affected by viscous heating $\widehat{Q}_{\text {vis }}$ (representing the energy dissipation by shock waves), radiative heating/cooling $\widehat{Q}_{\text {rad }}$ and hydrodynamical work $^{9} \widehat{Q}_{\mathrm{adb}}=-p \mathrm{~d} V / \mathrm{d} t$. After the passage of a shock wave with respective dissipative shock heating, the gas cools down quickly and relaxes towards radiative equilibrium (RE), eventually assisted by adiabatic cooling. This usually happens in a thin zone behind the shock fronts, extending between a few $1 / 1000 R_{\star}$ (unresolved in this case ${ }^{10}$ ) to about $1 / 10 R_{\star}$, depending on the pre-shock gas density. However, the details of the temperature structure in the post-shock relaxation zone are biased due to the usage of artificial viscosity. A more exact description of these structures and the relevant radiative cooling processes can be found in Woitke (2002). The gas in most parts of the outer atmosphere is hence characterised by an energetic equilibrium where the adiabatic cooling in the expanding wind and the re-expanding flow behind the compressing shock waves is balanced by the radiative heating due to absorption of radiation, in particular in ro-vibrational bands of polar molecules like $\mathrm{CO}$, henceforth called quasi-RE.

There are, however, also exceptions from this rule. (i) The dissociation/re-formation of $\mathrm{H}_{2}$ consumes/liberates so much energy (see Fig. 2) that the radiative relaxation is slowed down considerably, leading to parts in the model where the gas is out of RE and the mean molecular weight changes slowly in time. We call this effect the $\mathrm{H}_{2}$ energy buffer and discuss it further in Sect. 5.3. (ii) Inside of a newly forming (optically thick) dust shell, the radiative equilibrium temperature $T_{\text {rad }}$ can increase so quickly due to backwarming that the gas temperature cannot follow, thus causing this region to be temporarily out of RE. (iii) The gas density can be so small in the outermost regions (e.g. $n_{\langle\mathrm{H}\rangle} \lesssim 10^{5} \mathrm{~cm}^{-3}$ at $\gtrsim 20 R_{0}$ ) that the radiative heating/cooling rates become negligible and the gas

\footnotetext{
${ }^{9}$ Referred to as "adiabatical heating", therefore $\widehat{Q}_{\mathrm{adb}}$.

10 The width of the complete radiative relaxation zone is smaller than the grid spacing in this case, resulting in $T_{\mathrm{g}} \approx T_{\text {rad }}$ even at points where $\widehat{Q}_{\text {vis }}>0$, e.g. at the positions of the shock waves.
} 


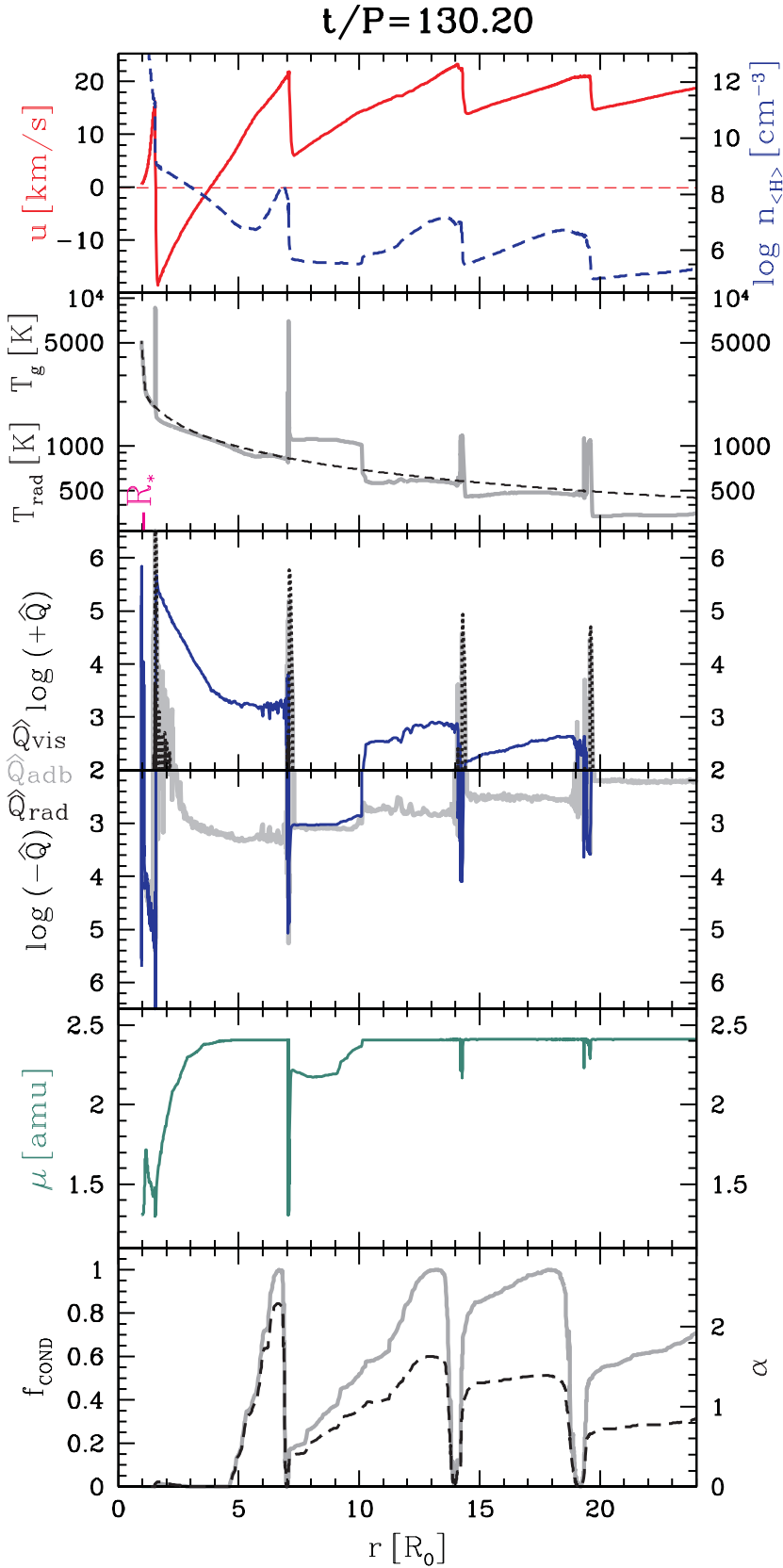

Fig. 6. A typical radial snapshot of a model with parameters $M_{\star}=$ $1 M_{\odot}, L_{\star}=4760 L_{\odot}, T_{\star}=3000 \mathrm{~K}, \mathrm{C} / \mathrm{O}=1.8, \Delta u=2 \mathrm{~km} \mathrm{~s}^{-1}$ and $P=310 \mathrm{~d}$ (derived quantities $R_{0}=1.78 \times 10^{13} \mathrm{~cm}$ and $\log g=-0.38$ ). Upper box: velocity $u$ (full line) and total hydrogen particle density $n_{\langle\mathrm{H}\rangle}$ (dashed). Second box: gas temperature $T_{\mathrm{g}}$ (full) and grey radiative equilibrium temperature $T_{\text {rad }}$ (dashed). Middle double box: radiative (full black), adiabatic (full grey) and viscous (dotted) heating rates (upper part) and cooling rates (lower part) in $[\mathrm{erg} / \mathrm{g} / \mathrm{s}]$. Fifth box: mean molecular weight $\mu$. Lower box: degree of condensation of carbon $f_{\text {cond }}$ (full) and ratio of radiative acceleration to gravitational deceleration $\alpha$ (dashed).

behaves almost adiabatically, usually resulting in lower temperatures in the expanding wind.

A general increase of the mean molecular weight from $\approx 1.3$ to $\approx 2.4$ can be observed in relaxed models, typically between the stellar radius and a distance of $\approx 5 R_{\star}$. This means that the gas in the outer atmosphere is often close to the dissociation equilibrium of $\mathrm{H}_{2}$ due to energetic constraints, i.e. $\mathrm{H}_{2}$ molecules are again and again destroyed by the propagating shocks and do only slowly form again. This behaviour is related to the dust formation which occurs at similar temperatures. It also suppresses the appearance of very low temperatures close to the star in consequence of a two-step cooling process (radiative + adiabatic) as it has been reported to occur in models for R Coronae Borealis stars (Paper II), which consist of a hydrogen-poor gas (mainly $\mathrm{He}$ and $\mathrm{C}$ ).

The depicted model is an example for a stellar wind with a relatively small mass loss rate $\left(\dot{M}=2.2 \times 10^{-7} M_{\odot} /\right.$ yr $)$. Even for such a thin circumstellar environment, the model does not show a long-lasting increase of the gas temperatures close to the star as consequence of the energy input by the stellar pulsation (the formation of a "calorisphere" as termed by Willson 2000). The radiative cooling rates are just to strong to allow for this effect. Consequently, we did not find any evidence for a purely pulsation-driven outflow in our model calculations - the models always reveal a mainly dust-driven wind, just as in the classical models.

\subsection{The $\mathrm{H}_{2}$-energy buffer}

Figure 7 shows three snapshots of a slow, non-dissociative shock wave propagating through the dust formation zone which is previously $\mathrm{H}_{2}$-rich. The radiative heating/cooling rates at post-shock densities $n_{\langle\mathrm{H}\rangle} \approx 10^{11} \ldots 10^{12} \mathrm{~cm}^{-3}$ are so efficient that the radiative cooling zone of this shock is unresolved (see Sect. 5.2) in the model, leading to an apparently smooth temperature structure around the shock front in the first two depicted time steps. Hence, in the dust formation region, the gas approximately behaves like in the isothermal limiting case, which probably explains why the general results of the new models are so similar to the classical ones. The compression by this $10 \mathrm{~km} \mathrm{~s}^{-1}$ shock wave initiates dust formation at about $1.7 R_{0}$ in the model, leading to a narrow peak in the degree of condensation (first depicted time step, lower box) and a remarkable step in the temperature structure by backwarming (second time-step, second box). Thereby, the shock wave does not only initiate dust formation, but also destroys most of the $\mathrm{H}_{2}$ simultaneously.

According to our dynamical model calculations, the potential energies stored in the bindings of $\mathrm{H}_{2}$-molecules play an important role for the thermal behaviour of the gas in the circumstellar environment of AGB stars. The dissociation/formation of $\mathrm{H}_{2}$ consumes/liberates so much energy that the gas temperature remains nearly constant during the phase transition between $\mathrm{H}$ and $\mathrm{H}_{2}$ (while the gas is constantly heated/cooled) ${ }^{11}$.

\footnotetext{
11 The internal chemical processes responsible for the formation of $\mathrm{H}_{2}$ are assumed to be very fast in our model (assumption of chemical equilibrium, see Sect. 3.1). Thereby, the actual speed of the $\mathrm{H}_{2}$-formation is limited by the need of the gas to radiate away the liberated binding energy in our model. It remains an open question whether the three-body gas phase reactions and the surface reactions on dust grains required for the kinetical formation of $\mathrm{H}_{2}$ are actually fast enough to achieve this limiting case. A kinetic treatment of the $\mathrm{H}_{2}$-formation would be desirable, but goes beyond the scope of this paper.
} 

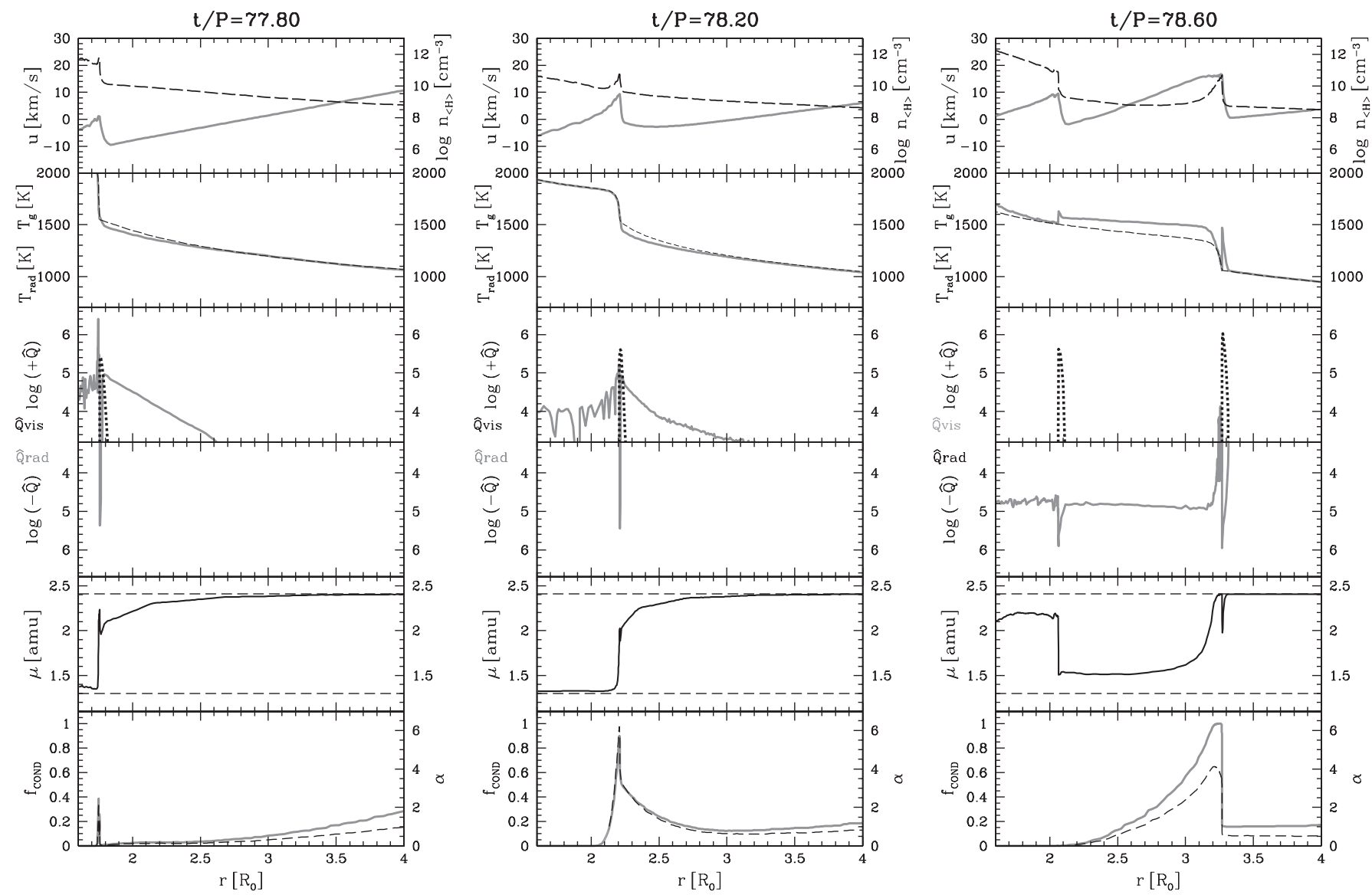

Fig. 7. Enlarged hydrodynamic structures of a dust-forming, non-dissociative shock wave propagating outwards. Parameters: $M_{\star}=1 M_{\odot}$, $L_{\star}=7500 L_{\odot}, T_{\star}=2600 \mathrm{~K}, \mathrm{C} / \mathrm{O}=1.8, \Delta u=2 \mathrm{~km} \mathrm{~s}^{-1}$ and $P=650 \mathrm{~d}$ (derived quantities $R_{0}=2.97 \times 10^{13} \mathrm{~cm}$ and $\log g=-0.82$ ). The plotted quantities are explained in the caption of Fig. 6 . For clarity, the adiabatic work is not depicted here.

This slow temperature increase/descrease can be interpreted in terms of an enlargement of the heat capacity of the transition state (see Fig. 2 and Eq. (24)), since the time-scale for radiative relaxation is proportional to the heat capacity as

$\tau_{\mathrm{rad}}=\frac{c_{\mathrm{V}}\left(\rho, T_{\mathrm{g}}, T_{\mathrm{rad}},\left\langle\frac{\mathrm{d} v}{\mathrm{~d} l}\right\rangle\right)}{\widehat{Q}_{\mathrm{rad}}\left(\rho, T_{\mathrm{g}}, T_{\mathrm{rad}},\left\langle\frac{\mathrm{d} v}{\mathrm{~d} l}\right\rangle\right)}\left(T_{\mathrm{rad}}-T_{\mathrm{g}}\right)$.

The last depicted time step in Fig. 7 shows the energy buffering effect in both directions. On the one hand, it limits the temperature increase by dissipative heating in the shock front. On the other hand, it delays the post-shock cooling of the gas ${ }^{12}$. By comparing the difference between $T_{\mathrm{g}}$ and $T_{\mathrm{rad}}$ with the mean molecular weight $\mu$ in Fig. 7, it becomes obvious that the state of quasi-RE ( $T_{\mathrm{g}} \approx T_{\mathrm{rad}}$ ) can often be reached only in those regions where hydrogen is predominantly atomic or predominantly molecular. The delaying influence of the $\mathrm{H}_{2}$-buffer can result in $\mathrm{H}_{2}$-forming regions staying out of $\mathrm{RE}$ for a substantial fraction of the pulsational period (see last depicted time step in Fig. 7 and central region around $8 R_{0}$ in Fig. 6).

\footnotetext{
12 Strictly speaking, this is not the post-shock-cooling here. What we observe is rather a quick decrease of $T_{\text {rad }}$ due to the decreasing backwarming by the outward propagating and thereby radially diluting dust-shell.
}

\subsection{Two-step cooling behind shock waves}

Figure 8 shows three consecutive snapshots of a dissociative shock wave propagating into a $\mathrm{H}_{2}$-rich gas. The parameters of this model describe a more compact AGB star than in Fig. 7, which results in stronger shock waves in the circumstellar environment ${ }^{13}$. The velocity of the considered shock wave is just above the dissociative threshold, resulting in post-shock temperatures between $5000 \mathrm{~K}$ and $7000 \mathrm{~K}$. We have divided each plot in Fig. 8 into five zones (by the vertical grey solid lines) to facilitate the identification of the different radiative and chemical processes.

- Zone 1 is the cool, $\mathrm{H}_{2}$-rich pre-shock gas in quasi-RE, which is not yet affected by the approaching shock wave and irrelevant for our discussion here.

- Zone 2 is the shock front, where the kinetic energy of the flow is mainly converted into internal energy of the gas by dissipative processes. This zone is numerically broadened by the usage of artificial viscosity, which gives approximately the right amount of dissipated energy, but the energy input is unphysically smeared out over this zone. Therefore, we refrain from any discussion of this zone.

13 The shock wave velocity is rather controlled by $\log g$ and $P$ than by $\Delta u$, compare footenote 2 in Paper II. 

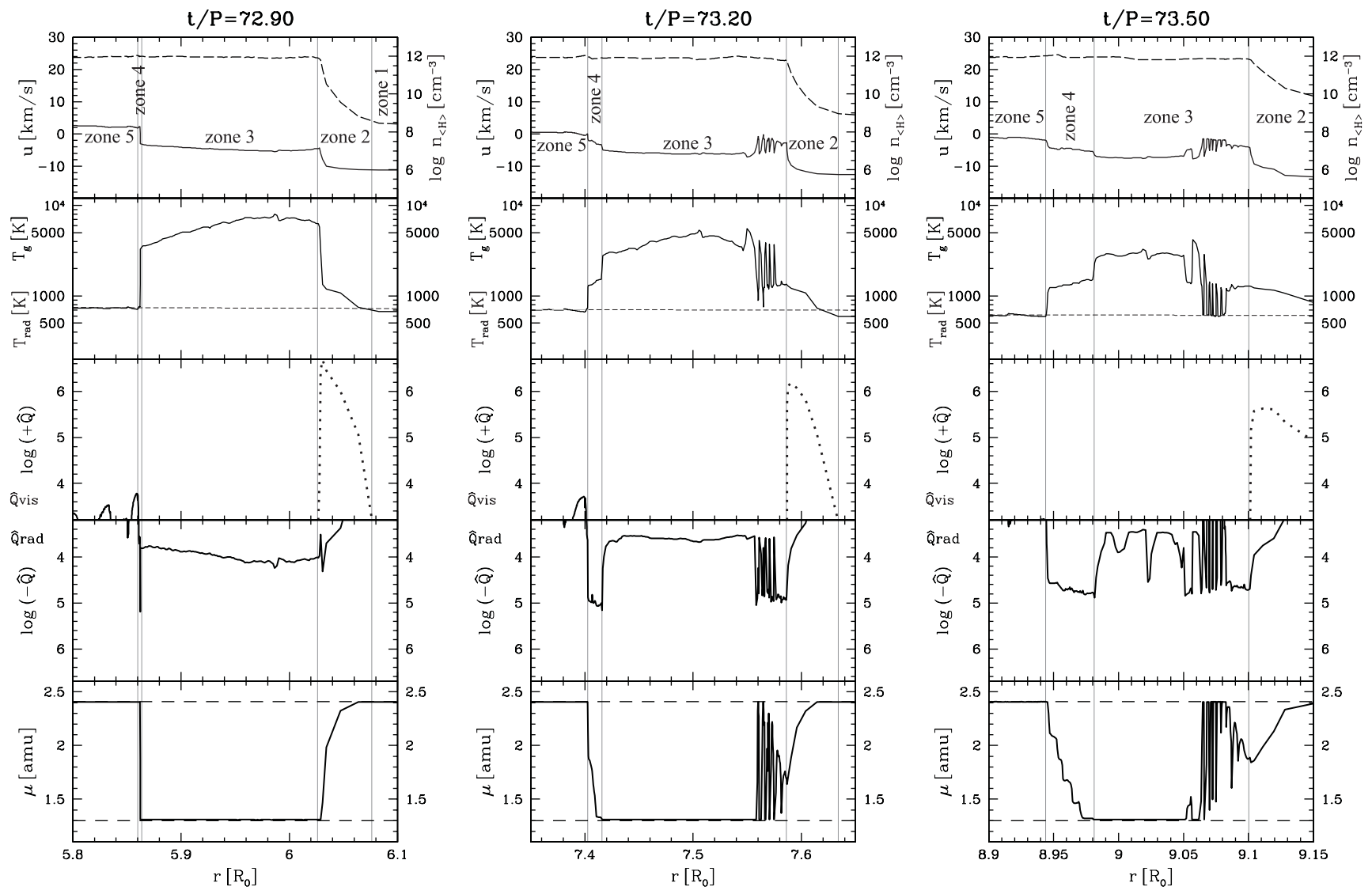

Fig. 8. A dissociative shock wave propagating through the circumstellar gas. Parameters: $M_{\star}=1.2 M_{\odot}, L_{\star}=5850 L_{\odot}, T_{\star}=2600 \mathrm{~K}, \mathrm{C} / \mathrm{O}=1.8$, $\Delta u=2 \mathrm{~km} \mathrm{~s}^{-1}$ and $P=650 \mathrm{~d}$ (derived quantities $R_{0}=2.62 \times 10^{13} \mathrm{~cm}$ and $\log g=-0.64$ ). The plotted quantities are explained in the caption of Fig. 6, however, the velocity is depicted as dashed line and the density as full line here.

- Zones 3 and 4 are the actively cooling post-shock regions with vanishing influence of the artificial viscosity $\widehat{Q}_{\text {vis }}=0$.

- Zone 4 is featured by a re-increase of the radiative cooling rate $\widehat{Q}_{\mathrm{rad}}$.

- In zone 5, the gas is back in quasi-RE.

All molecules in zone 3 are dissociated, but the gas temperature is with $3000 \mathrm{~K}$ to $6000 \mathrm{~K}$ not high enough to collisionally excite permitted cooling lines like $\mathrm{Ly} \alpha, \mathrm{H} \alpha$, Ca II $\mathrm{H}+\mathrm{K}$ or $\mathrm{Mg}$ II h+k. However, some low-lying energy levels of certain forbidden and fine-structure lines, in particular of Fe I and Fe II, can still be excited (Woitke \& Sedlmayr 1999). Consequently, the mainly neutral atomic gas cools slowly by radiative losses of the order of $10^{3} \ldots 10^{4} \mathrm{erg} \mathrm{g}^{-1} \mathrm{~s}^{-1}$ (at densities $n_{\langle\mathrm{H}\rangle} \approx 10^{7} \mathrm{~cm}^{-3}$ ).

This moderate radiative cooling is sufficient to reach temperatures $\$ 3000 \mathrm{~K}$ appropriate for molecule formation within a distance of about $0.1 R_{0}$ to $0.2 R_{0}$ behind the shock front. Once the first molecules are present (zone 4), the large amount of permitted ro-vibrational lines of polar molecules, in particular $\mathrm{CO}$, causes a substantial re-increase of the radiative cooling rate (by about one order of magnitude in the depicted case, despite the fact that the difference between $T_{\mathrm{g}}$ and $T_{\text {rad }}$ is here at least $2000 \mathrm{~K}$ smaller than in zone 3 ). From the mean molecular weight plotted in the lower panel in Fig. 8, we see that zone 4 is also the site where the re-formation of $\mathrm{H}_{2}$ takes place. In fact, the re-increase of the cooling rate (by CO-formation) occurs slightly before the re-formation of $\mathrm{H}_{2}$ begins. The radiative cooling in zone 4 is at first prolonged by the $\mathrm{H}_{2}$-energybuffer. Once the re-formation of $\mathrm{H}_{2}$ is complete, however, an almost instantaneous relaxation towards quasi-RE results and zone 5 is reached.

Since the gas pressure in the (sub-sonic) downstream gas (zones 3-5) remains approximately constant, the phases of efficient radiative cooling are accompanied by a compression of the gas. Accordingly, the upper panels of Fig. 8 show that the initial shock-compression is followed by another phase of postshock compression in zone 4.

\subsection{A radiative instability}

The second and third depicted time step in Fig. 8 show a strongly wriggled region concerning the spatial structure of $T_{\mathrm{g}}$, $\rho, \widehat{Q}_{\text {rad }}$ and $\mu$ at the right edge of zone 3 , which develops from the "smooth" structure on the 1.h.s. as time passes. After checking the numerics ${ }^{14}$, we conclude that this feature is actually

\footnotetext{
${ }^{14}$ Neither a variation of the resolution, nor of the averaging procedure for the velocity gradient $\left\langle\frac{\mathrm{d} v}{\mathrm{~d} l}\right\rangle$ resulted in a qualitative change of this cooling behaviour. Of course, we cannot exclude an influence of the numerical method on these results. However, even if the initial temperature pertubations are of numerical origin, the amplification mechanism clearly has a physical nature.
} 
caused by a physical effect which we identify as a radiative instability of the post-shock cooling gas.

The main effect is the re-increase of the cooling rate with decreasing gas temperature as described in Sect. 3.2. This causes the cooling trajectories in the post-shock region to be unstable. If parts of a cooling volume have already reached slightly lower temperatures, the further cooling of these parts is faster and the temperature difference to the other, still warmer parts in the volume increases exponentially.

The main effect is schematically depicted on the r.h.s. of Fig. 9. An increase of the radiative cooling rate $-\widehat{Q}_{\text {rad }}$ leads to a temperature decrease $T_{\mathrm{g}}(\ominus)$ which causes an increase of the molecular concentrations $c_{\text {mol }}(\ominus)$ which accelerates the cooling $(\oplus)$. This leads to an overall positive feedback in the r.h.s. control loop $(\ominus \cdot \ominus \cdot \oplus=\oplus)$ which means that small perturbations in the system are self-amplifying and can grow to large temperature differences, which characterises this instability. Another control loop is depicted on the 1.h.s. of Fig. 9. The post-shock cooling gas is approximately in pressure balance, because the downstream flow is subsonic (close inspection of Fig. 9 in fact shows that the temperature and density wriggles are anti-correlated wheras the pressure structure is smooth). Therefore, a decrease of the temperature $T_{\mathrm{g}}$ causes an increase of the density $\rho(\ominus)$ which increases the collision rates what usually (compare Fig. 3) has a positive effect on the cooling rate $-\widehat{Q}_{\text {rad }}(\oplus)$ which decreases the temperature $(\ominus)$ - another unstable mechanism which coworks with the former control loop.

The impact of this instability on our calculations can be seen in Fig. 8. The initially small pertubations of the gas temperature $T_{\mathrm{g}}$ (probably of numerical nature, see 1.h.s. of Fig. 8) are amplified and result in a very inhomogeneous hydrodynamic structure, where cool, fast cooling and dense domains alternate with comparatively hot, slow cooling and thin domains. Density inhomogeneities of about one order of magnitude are generated which, however, finally disappear when all cooling domains have reached quasi-RE. Since the spatial extentions of the inhomogeneities are of the order of the grid spacing (a single peak consists of 1-4 Lagrangian elements), we conclude that we are not able to spatially resolve the developing structures.

In our models, the described radiative cooling instability occurs if the following criteria are fulfilled:

- The energy dissipation in the shock wave is strong enough to completely dissociate all molecules relevant for the radiative cooling (in particular $\mathrm{CO}$ );

- The shocked gas remains in pressure equlibrium with its local environment;

- The mass density of the shocked gas does not exceed a value of $\approx 10^{-13} \mathrm{~g} / \mathrm{cm}^{3}$, otherwise the effect of increasing optical depth in the spectral lines inhibits the instability (see Fig. 3).

The described radiative instability is of course not restricted to spherical symmetry as our model, and the resulting structure formation in the post-shock cooling gas, temporarily leading to a side-by-side of molecule-rich and molecule-poor regions,

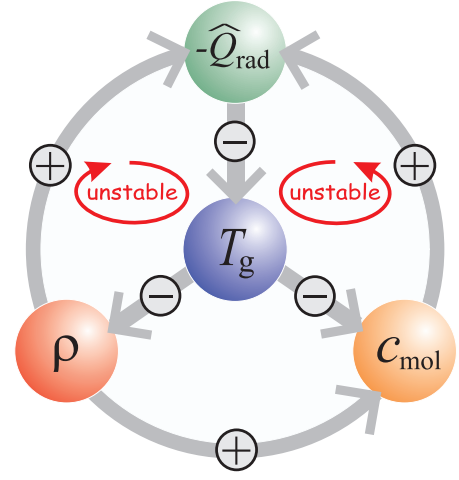

Fig. 9. Control loop of the radiative instability. $\oplus$ and $\ominus$ mark positive and negative feedbacks, respectively. For example, $\ominus$ indicates that an increase of the causing quantity results in a decrease of the affected quantity and vice versa. All closed loops (including the big circle) have an overall positive feedback, i.e. are self-amplifying.

may be part of the explanation of the internal "clumpiness" of the thin CO-shells observed in the detached shells of TTCyg by (Olofsson et al. 2000).

\section{Conclusions}

In this paper, dynamical model calculations for the winds of $\mathrm{C}$-stars have been presented which contain a time-independent non-LTE description of the gas and a time-dependent description of dust nucleation, growth and evaporation. The results have been obtained by applying the non-LTE state and cooling functions developed in Paper I in the frame of the Berlin CHILD-code developed by Fleischer et al. (1992). These new models are technically realised by using 4-dimensional tables of the state functions in the hydro-code. According to the outlined approximations for the non-LTE treatment of the gas, the state functions (pressure $p$, internal energy $e$, radiative net heating function $\left.\widehat{Q}_{\text {rad }}\right)$ depend on $\rho, T_{\mathrm{g}}, T_{\text {rad }}$ and $\left\langle\frac{\mathrm{d} v}{\mathrm{~d} l}\right\rangle$.

The main result of the new models is that the gas behaves almost isothermal close to the star, in particular in the dust formation zone, where the densities are of the order of $n_{\langle\mathrm{H}\rangle} \approx$ $10^{9} \ldots 10^{11} \mathrm{~g} \mathrm{~cm}^{-3}$. Just at these densities, the efficiency of the energy exchange between gas and radiation field is maximum which results in a close coupling of the gas to the condition of radiative equilibrium (RE). Therefore, propagating shock waves caused by the stellar pulsation are only accompanied by very narrow post-shock cooling zones in this region (actually unresolved in our numerical models).

Consequently, the dust formation and wind acceleration proceeds in a very similar way as outlined by Fleischer et al. (1992) and Höfner et al. (1995) and our conclusions about the mass loss mechanism of AGB stars are basically identical. We generally find a dust-driven outflow triggered by the pulsation of the star. In contrast, no computational evidence for a longlasting increase of the temperatures close to the pulsating AGB star have been found in the models (the occurrence of a "calorisphere" as termed by Willson 2000), which is a straightforward consequence of the applied radiative cooling rates. Only if the pre-shock gas densities were as small as $n_{\langle\mathrm{H}\rangle} \lesssim 10^{7} \mathrm{~cm}^{-3}$, the radiative cooling would be so inefficient that the relaxation 
towards RE would remain incomplete, before the next shock wave encounters the gas element. However, such small gas densities close to the star are not consistent with the observed mass loss rates, which implies that the purely pulsation-driven mass loss as proposed by Bowen (1988) is incompatible with our non-LTE radiative cooling rates.

The new model calculations have recently been used as reference model for the analysis of permitted and forbidden Fe II emission lines as observed in Mira stars (Richter \& Wood 2001; Richter et al. 2002). The non-LTE line transfer calculations performed on detailed shock structures, resulting from the application of our radiative cooling rates in thermodynamical models of periodically shocked fluid elements, suggest that the narrow but hot post-shock cooling zones, as occurring in the presented dynamical model calculations, are sufficient to explain the observed Fe II emission line fluxes.

There occur, however, also several new features in the models which are caused by the introduction of the non-LTE state functions and have not been reported so far. These effects are generally more pronounced outside of the dust formation zone, where the densities (and hence the radiative cooling rates) are smaller.

- The varying molecular weight $\mu$ plays an important role for the structure of the photosphere and the pressuredominated regions close to the star, in particular concerning the hydrostatic start structure. In comparison to a monoatomic gas, the refined thermal equation of state usually results in a steeper hydrostatic density gradient.

- The inclusion of the binding energies of $\mathrm{H}_{2}$-molecules in the caloric equation of state has a significant influence on the thermal behaviour of the gas in the transition region $\mathrm{H} \rightleftharpoons \mathrm{H}_{2}$ close to the star as well as on the response of the gas to the energy dissipation caused by propagating shock waves.

- Dissociative shock waves occur for shock velocities $\gtrsim 20 \mathrm{~km} \mathrm{~s}^{-1}$ (see also Richter et al. 2002). Only these shocks are energetically capable to destroy all $\mathrm{H}_{2}$ molecules and are characterised by high post-shock temperatures, typically $8000 \mathrm{~K}$ to $11000 \mathrm{~K}$ and extended post-shock cooling zones. In contrast, weaker shock waves produce much less pronounced temperature peaks.

- In combination with the much smaller non-LTE radiative cooling rates as compared to LTE considerations, the $\mathrm{H}_{2}$-reformation significantly delays the radiative relaxation towards RE by the liberation of chemical binding energies. This effect (the " $\mathrm{H}_{2}$ energy buffer") may result in extended regions at a distance of several stellar radii away from the star that remain in non-RE for a considerable fraction of one pulsational period. This behaviour might be related to the physical origin of the so-called warm molecular layers (Tsuji et al. 1997).

- The formation of molecules in the radiative relaxation zone behind a dissociative shock wave leads to an acceleration of the cooling process. Thus, a two-step cooling is typical where the initially fast cooling by permitted atomic lines down to $\approx 6000 \mathrm{~K}$ is followed by a second phase of intense molecular cooling below $\approx 3000 \mathrm{~K}$, accompanied by a second phase of post-shock compression. In between, the gas mainly cools by forbidden metal emission lines, or adiabatically.

- The re-increase of the radiative cooling rates with decreasing gas temperature due to molecule formation causes a radiative instability of the post-shock cooling gas at small densities. This instability may lead to a spatial coexistence of cool molecule-rich and warm molecule-poor regions in the radiative relaxation zone behind dissociative shock waves, possibly related to the internal clumpiness of the detached shells of TT Cyg as observed by (Olofsson et al. 2000).

Another remarkable point is that in our models, the $\mathrm{H}_{2}$ formation and the dust formation are closely related due to energetic constraints (the $\mathrm{H}_{2}$ energy buffer). A dynamic treatment of the process of $\mathrm{H}_{2}$ formation by three-body gas phase reactions and surface reactions on dust grains would, therefore, be highly desirable.

Acknowledgements. We want to thank Jan Martin Winters and Axel Fleischer for providing us with the CHILD-CODE, thus making this work possible. Furthermore we want to thank Thorsten Arndt for many valuable advices. This work has been supported by the DFG, Sonderforschungsbereich 555, Komplexe Nichtlineare Prozesse, part project B8. The numerical tables of the non-LTE state functions have been computed on the T3E parallel computer at the Konrad-ZuseZentrum für Informationstechnik Berlin, project bvpt17.

\section{Appendix A: Non-LTE treatment of molecules}

The contribution of ro-vibrational line transitions of molecules to the net radiative heating rate $\widehat{Q}_{\text {rad }}$ is calculated by means of an approximate non-LTE description of their ro-vibrational states. The level energies are assumed to be given by $E(v, J)$ where $v$ and $J$ are appropriate sets of quantum numbers describing the vibrational and rotational state of the molecule, the structure of which may vary with the type of the molecule.

As in Paper I, we introduce two yet unknown excitation temperatures $T_{\text {vib }}, T_{\text {rot }}$ for each molecule by assuming

$n_{v, J}=n_{\mathrm{mol}} \frac{g_{J} g_{v}}{Z_{\mathrm{vib}} Z_{\mathrm{rot}}} \exp \left(-\frac{E_{\mathrm{vib}}(v)}{k T_{\mathrm{vib}}}-\frac{E_{\mathrm{rot}}(v, J)}{k T_{\text {rot }}}\right)$

$n_{\text {mol }}$ is the total particle density of the molecule and $n_{v, J}$ the particle density in the state $(v, J) . E_{\mathrm{vib}}(v)=E(v, 0)-E(0,0)$ is the vibrational excitation energy in the rotational ground state and $E_{\mathrm{rot}}(v, J)=E(v, J)-E(v, 0)$ the rotational excitation energy. $Z_{\mathrm{vib}}, Z_{\mathrm{rot}}$ and $g_{J}, g_{v}$ are the vibrational and rotational partition functions and the statistical weights, respectively.

The drawback of the methods developed in Paper I was that the calculations were restricted to simple types of molecules (diatomic or linear) and simple types of transitions (pure rotational and fundamental mode vibrational). Furthermore, $T_{\text {rot }}$ and $T_{\text {vib }}$ were calculated independently, which is physically not correct, because the ro-vibrational line transitions affect both. In this appendix, we aim at a more general formulation of the problem where we can account for arbitrary types of molecules and can handle pure rotational and ro-vibrational transitions of 
various type (also overtone transitions and combination bands) simultaneously.

The unknown excitation temperatures $T_{\text {vib }}, T_{\text {rot }}$ are determined by considering the gains and losses of vibrational and rotational excitation energies of the molecule as caused by radiative and collisional transitions. For the radiative transitions (r.h.s. of Fig. A.1), the energy of each absorbed or emitted photon is split into

$h v=\Delta E_{\mathrm{vib}}+\Delta E_{\mathrm{rot}}$,

where the energy differences are $\Delta E_{\mathrm{vib}}=E_{\mathrm{vib}}\left(v_{u}\right)-E_{\mathrm{vib}}\left(v_{\ell}\right)$ and $\Delta E_{\mathrm{rot}}=E_{\mathrm{rot}}\left(v_{u}, J_{u}\right)-E_{\mathrm{rot}}\left(v_{\ell}, J_{\ell}\right)$. Introducing lower and upper sets of ro-vibrational quantum numbers as $u=\left(v_{u}, J_{u}\right)$ and $\ell=$ $\left(v_{\ell}, J_{\ell}\right)$ for each line transition, the net radiative energy transfer rates to the vibrational and rotational excitation are given by

$Q_{\mathrm{rad}}^{\mathrm{vib}}=\sum_{\text {lines }} \Delta E_{\mathrm{vib}} A_{u \ell} P_{u \ell}^{\mathrm{e}}\left(n_{\ell} \frac{g_{u}}{g_{\ell}} j_{v_{u \ell}}-n_{u}\left(1+j_{v_{u \ell}}\right)\right)$

$Q_{\mathrm{rad}}^{\mathrm{rot}}=\sum_{\text {lines }} \Delta E_{\mathrm{rot}} A_{u \ell} P_{u \ell}^{\mathrm{e}}\left(n_{\ell} \frac{g_{u}}{g_{\ell}} j_{v_{u \ell}}-n_{u}\left(1+j_{v_{u}}\right)\right)$

with the Einstein-coefficient $A_{u \ell}$, the escape probability $P_{u \ell}^{\mathrm{e}}$ and a dimensionless form of the continuous background intensity $j_{v}=c^{2} /\left(2 h v^{3}\right) J_{v}^{\text {cont }}$ as introduced in Paper I.

For the collisional processes (1.h.s. of Fig. A.1), the same splitting of energy of general type $\left(v^{\prime}, J^{\prime}\right) \rightarrow\left(v^{\prime \prime}, J^{\prime \prime}\right)$ could be carried out, too. However, the cross sections for such general collision rates is usually not available. Instead we consider collisions which only change the rotational or the vibrational state of the molecule. The respective collisional energy transfer rates are calculated as

$Q_{\text {coll }}^{\mathrm{vib}}=\sum_{v^{\prime}>v^{\prime \prime}} \Delta E_{\mathrm{vib}} C_{v^{\prime} v^{\prime \prime}}\left(n_{v^{\prime}}-n_{v^{\prime \prime}} \frac{g_{v^{\prime}}}{g_{v^{\prime \prime}}} \mathrm{e}^{-\frac{\Delta E_{\mathrm{vib}}}{k T g}}\right)$

$Q_{\text {coll }}^{\text {rot }}=\sum_{J^{\prime}>J^{\prime \prime}} \Delta E_{\text {rot }} C_{J^{\prime} J^{\prime \prime}}\left(n_{J^{\prime}}-n_{J^{\prime \prime}} \frac{g_{J^{\prime}}}{g_{J^{\prime \prime}}} \mathrm{e}^{-\frac{\Delta E_{\text {rot }}}{k T_{\mathrm{g}}}}\right)$

where now $\Delta E_{\mathrm{vib}}=E_{\mathrm{vib}}\left(v^{\prime}\right)-E_{\mathrm{vib}}\left(v^{\prime \prime}\right)$ and $\Delta E_{\mathrm{rot}}=E_{\mathrm{rot}}\left(0, J^{\prime}\right)-$ $E_{\text {rot }}\left(0, J^{\prime \prime}\right), n_{v}$ is the total occupational number of the molecule in the vibrational state $v$ (sum over rotational sub-states) and $n_{J}$ the total occupational number of the molecule in the rotational state $J$ (disregarding vibrational excitation here).

In statistical equilibrium, the occupational numbers of all $(v, J)$-states have relaxed toward a steady state. Consequently, the energies contained in the molecule in form of rotational and vibrational excitation are constant, i.e.

$Q_{\mathrm{rad}}^{\mathrm{vib}}=Q_{\mathrm{coll}}^{\mathrm{vib}} \quad$ and $\quad Q_{\mathrm{rad}}^{\mathrm{rot}}=Q_{\mathrm{coll}}^{\mathrm{rot}}$.

The steady state condition (A.7) provides two non-linear coupled algebraic equations for the two unknowns $T_{\text {vib }}$ and $T_{\text {rot }}$, which can be solved by standard iterative numerical methods. Finally, the total contribution of the molecule to the net radiative heating rate of the gas is given by

$Q_{\mathrm{rad}}(\mathrm{mol})=Q_{\mathrm{rad}}^{\mathrm{vib}}+Q_{\mathrm{rad}}^{\mathrm{rot}}=Q_{\mathrm{coll}}^{\mathrm{vib}}+Q_{\mathrm{coll}}^{\mathrm{rot}}$.

$Q_{\text {rad }}(\mathrm{mol})$ can be optionally calculated from the 1.h.s. or the r.h.s. of Eq. (A.8), a property which can be used to check

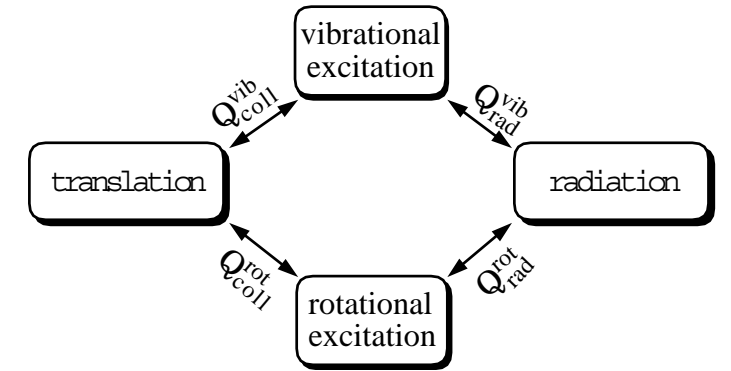

Fig. A.1. Energy fluxes between the internal energy of the gas contained in its translational degrees of freedom, a molecule subdivided into internal vibrational and rotational excitation energies, and the radiation field.

the quality of the solution. According to Eq. (A.8), the rovibrational states of molecules act only as a transmitter, transferring radiative energy into thermal kinetic energy (radiative heating) or vice versa (radiative cooling) in statistical equilibrium.

Detailed level energies and transition probabilities for $\mathrm{H}_{2} \mathrm{O}$, $\mathrm{HCN}, \mathrm{C}_{2} \mathrm{H}_{2}, \mathrm{~N}_{2}, \mathrm{CO}_{2}, \mathrm{H}_{2} \mathrm{~S}, \mathrm{SO}_{2}, \mathrm{OH}$ and OCS have been deduced from the HITRAN-database (Rothman et al. 1987) as summarised in Table A.2. The radiative data for $\mathrm{H}_{2}$ are taken from Turner et al. (1977). The vibrational deexcitation rates due to collisions with $\mathrm{H}, \mathrm{He}$ and $\mathrm{H}_{2}$ to the vibrational ground state $C_{v^{\prime} 0}$ are generally calculated by means of the analytical formula of Millikan \& White (1964) (see Eq. (41) in Paper I), except for $\mathrm{CO}$ (Neufeld \& Hollenbach 1994) and for $\mathrm{H}_{2}$ (Lepp \& Shull 1983). In case of the molecules listed in Table A.2, we only take into account these collision rates by considering the fundamental frequencies of the molecules. In all other cases (simple diatomic molecules), also the collisional deexcitation rates to excited vibrational levels $C_{v^{\prime} v^{\prime \prime}}$ are taken into account, using "surprisal analysis" (Elitzur 1983, see Eq. (44) in Paper I).

The net energy transfer rate to the rotational excitation energy due to collisions with $\mathrm{H}, \mathrm{H}_{2}$ and $\mathrm{He}$, can be summarised as stated in Eq. (35) of Paper I. Concerning the molecules listed in Table A.2, the total rotational cross section $\sigma_{0}$ is thereby scaled with the measured value for $\mathrm{H}_{2} \mathrm{O}$ (Hollenbach \& McKee 1989) according to the "size" of the molecule (estimated from the covolume $V_{\mathrm{c}}$ in the Van-der-Waals law, data from Weast et al. 1989)

$\sigma_{0}(\mathrm{~mol})=1.5 \times 10^{-16} \mathrm{~cm}^{2}\left(\frac{V_{\mathrm{c}}(\mathrm{mol})}{V_{\mathrm{c}}\left(\mathrm{H}_{2} \mathrm{O}\right)}\right)^{2 / 3}$.

For some important polar diatomic molecules, we follow a different strategy. Detailed level energies for $\mathrm{CO}, \mathrm{SiO}$ and $\mathrm{CS}$ and the radiative data for their fundamental mode, 1 st and 2 nd overtone transitions have been kindly provided by Jørgensen (1997, priv. comm.). From these data, we fit $E(v, J)\left[\mathrm{cm}^{-1}\right]$ and the Einstein coefficients $A_{u \ell}\left[\mathrm{s}^{-1}\right]$ separately for each $p$ 
Table A.1. Fit coefficients for ro-vibrational energy levels and Einstein coefficients for polar diatomic molecules.

\begin{tabular}{|c|c|c|c|c|c|c|c|}
\hline & $\omega_{\mathrm{e}}$ & $\omega_{\mathrm{e}} x_{\mathrm{e}}$ & $\omega_{\mathrm{e}} y_{\mathrm{e}}$ & $B_{\mathrm{e}}$ & $a_{\mathrm{e}}$ & $D_{\mathrm{e}}$ & $b_{\mathrm{e}}$ \\
\hline $\mathrm{CO}$ & 2169.8028 & 13.298496 & 0.0119410 & 1.9311085 & 0.0173306 & $6.0415110(-6)$ & $4.7428669(-8)$ \\
\hline $\mathrm{SiO}$ & 1241.5269 & 5.9722051 & 0.0058210 & 0.7267607 & 0.0050236 & $9.8706042(-7)$ & $-1.6899730(-9)$ \\
\hline \multirow[t]{2}{*}{$\mathrm{CS}$} & 1285.1403 & 6.5013679 & 0.0037048 & 0.8200434 & 0.0059208 & $1.3313737(-6)$ & $2.7598168(-9)$ \\
\hline & branch & $a_{1}$ & $a_{2}$ & $a_{3}$ & $a_{4}$ & $a_{5}$ & $a_{6}$ \\
\hline \multirow[t]{6}{*}{$\mathrm{CO}$} & (1a) & 35.126 & $-2.5964(-3)$ & $3.3506(-4)$ & $9.9885(-1)$ & $-1.3553(-3)$ & $-7.7475(-6)$ \\
\hline & (1b) & 36.010 & $4.9628(-3)$ & $3.2473(-5)$ & $9.9355(-1)$ & $-6.5762(-3)$ & $-3.8480(-5)$ \\
\hline & (2a) & $1.3677(-1)$ & $-5.9457(-4)$ & $3.3151(-6)$ & 1.5155 & $5.5514(-1)$ & $7.5813(-3)$ \\
\hline & $(2 b)$ & $1.4572(-1)$ & $6.2297(-4)$ & $5.7196(-6)$ & 1.4993 & $5.4100(-1)$ & $2.8323(-3)$ \\
\hline & (3a) & $5.0317(-4)$ & $-4.4145(-6)$ & $2.8274(-8)$ & 2.7763 & $2.4531(-1)$ & $6.2720(-1)$ \\
\hline & $(3 b)$ & $6.4503(-4)$ & $4.0147(-6)$ & $1.0813(-7)$ & 2.3048 & $6.6627(-1)$ & $4.0540(-1)$ \\
\hline \multirow[t]{6}{*}{$\mathrm{SiO}$} & (1a) & 35.879 & $1.2589(-1)$ & $1.5964(-4)$ & $9.8628(-1)$ & $-4.6472(-3)$ & $-5.8526(-4)$ \\
\hline & (1b) & 36.410 & $-1.2753(-1)$ & $2.3829(-5)$ & $9.8033(-1)$ & $-9.2596(-3)$ & $-6.5078(-4)$ \\
\hline & (2a) & $1.8221(-1)$ & $3.9730(-5)$ & $1.6372(-6)$ & 1.4516 & $6.9366(-1)$ & $-7.0641(-3)$ \\
\hline & $(2 b)$ & $1.8450(-1)$ & $-3.3486(-5)$ & $1.0777(-6)$ & 1.4470 & $6.9103(-1)$ & $-9.0454(-3)$ \\
\hline & (3a) & $6.9651(-4)$ & $-4.0242(-7)$ & $-2.8118(-9)$ & 1.2052 & 4.9774 & $2.0843(-2)$ \\
\hline & $(3 b)$ & $7.5263(-4)$ & $1.6333(-6)$ & $-4.704(-10)$ & 1.3146 & 4.4637 & $3.5736(-2)$ \\
\hline \multirow[t]{6}{*}{$\mathrm{CS}$} & (1a) & 77.742 & $-1.1589(-1)$ & $3.4157(-4)$ & 1.0000 & $2.9834(-4)$ & $-1.8336(-4)$ \\
\hline & (1b) & 79.065 & $1.2039(-1)$ & $2.3319(-4)$ & $9.9767(-1)$ & $-2.1245(-3)$ & $-1.7985(-4)$ \\
\hline & $(2 a)$ & $2.4199(-1)$ & $-1.0460(-3)$ & $3.8244(-6)$ & 1.5266 & $5.2586(-1)$ & $1.2063(-2)$ \\
\hline & $(2 b)$ & $2.5370(-1)$ & $1.0547(-3)$ & $7.0820(-6)$ & 1.5175 & $5.2067(-1)$ & $8.2837(-3)$ \\
\hline & (3a) & $1.0563(-3)$ & $-7.8715(-6)$ & $2.8565(-8)$ & 2.2132 & $6.7195(-1)$ & $3.1164(-1)$ \\
\hline & (3b) & $1.1605(-3)$ & $7.8538(-6)$ & $8.5469(-8)$ & 2.0905 & $7.8194(-1)$ & $2.6466(-1)$ \\
\hline
\end{tabular}

(1a) fundamental mode ( $r$-branch): $v \rightarrow v-1, J \rightarrow J-1$. (1b) fundamental mode ( $p$-branch): $v \rightarrow v-1, J \rightarrow J+1$. (2a) 1st overtone ( $r$-branch): $v \rightarrow v-2, J \rightarrow J-1$. (2b) 1st overtone ( $p$-branch): $v \rightarrow v-2, J \rightarrow J+1$. (3a) 2nd overtone ( $r$-branch): $v \rightarrow v-3, J \rightarrow J-1$. (3b) 2nd overtone ( $p$-branch): $v \rightarrow v-3, J \rightarrow J+1$ for emission.

and $r$-branch to the $J<100$ and $v<10$ data (see Table A.1), using the following analytical representations

$$
\begin{aligned}
E(v, J)= & \omega_{\mathrm{e}}\left(v+\frac{1}{2}\right)-\omega_{\mathrm{e}} x_{\mathrm{e}}\left(v+\frac{1}{2}\right)^{2}+\omega_{\mathrm{e}} y_{\mathrm{e}}\left(v+\frac{1}{2}\right)^{3} \\
& +\left(B_{\mathrm{e}}-a_{\mathrm{e}}\left(v+\frac{1}{2}\right)\right) J(J+1) \\
& -\left(D_{\mathrm{e}}+b_{\mathrm{e}}\left(v+\frac{1}{2}\right)\right) J^{2}(J+1)^{2}
\end{aligned}
$$

$$
\begin{aligned}
A_{u \ell}=\frac{10^{-10}}{g_{u}}\left(\frac{v_{u \ell}}{c}\right)^{3} & \left(a_{1}\left(J^{\prime \prime}+1\right)+a_{2} J^{\prime \prime 2}+a_{3} J^{\prime \prime 3}\right) \\
& \times\left(1+a_{4} v_{\ell}+a_{5} v_{\ell}^{2}+a_{6} v_{\ell}^{3}\right)
\end{aligned}
$$

where $J^{\prime \prime}=\operatorname{Min}\left\{J_{u}, J_{\ell}\right\}$. These analytical formulae enable us to treat the radiative transitions more systematically, e.g. to adjust the necessary number of transitions according to the temperature $T_{\mathrm{g}}$ and the radiation field. Additionally, the pure rotational radiative transitions are included in the way described in Paper I. Other polar diatomic molecules $(\mathrm{OH}, \mathrm{CH}, \mathrm{CN}, \mathrm{SiS}$, $\mathrm{SiC}, \mathrm{SiN}, \mathrm{SO}, \mathrm{HS}$ ), which have turned out not to be very important for the radiative heating/cooling, are still included by their simplified energy levels and radiative data for the pure rotational and fundamental mode ro-vibrational transitions as described in Paper I.
Table A.2. Overview of the data obtained from the HITRAN-database for polyatomic molecules and $\mathrm{N}_{2}$, and for $\mathrm{H}_{2}$.

\begin{tabular}{lllll}
\hline \hline Molecule & Rot. states $^{1}$ & Vib. states & Lines & Co-volume \\
\hline $\mathrm{H}_{2}$ & 20 & 5 & 1012 & 0.02661 \\
$\mathrm{H}_{2} \mathrm{O}$ & 387 & 62 & 28171 & 0.03049 \\
$\mathrm{HCN}$ & 46 & 5 & 703 & 0.04 \\
$\mathrm{C}_{2} \mathrm{H}_{2}$ & 60 & 9 & 1426 & 0.05136 \\
$\mathrm{~N}_{2}$ & 41 & 2 & 120 & 0.03913 \\
$\mathrm{CO}_{2}$ & 55 & 110 & 27093 & 0.04267 \\
$\mathrm{H}_{2} \mathrm{~S}$ & 487 & 5 & 5108 & 0.04287 \\
$\mathrm{SO}_{2}$ & 1787 & 8 & 38532 & 0.05636 \\
$\mathrm{OCS}$ & 99 & 3 & 461 & 0.05817 \\
\hline
\end{tabular}

${ }^{1}$ In the vibrational ground state. Numbers of rotational states in excited vibrational levels are similar.

The total rotational cross sections for the diatomic molecules are scaled according to the measured value for $\mathrm{CO}$ (Hollenbach \& McKee 1979)

$\sigma_{0}(\mathrm{~mol})=3.0 \times 10^{-16} \mathrm{~cm}^{2}\left(\frac{V_{\mathrm{c}}(\mathrm{mol})}{V_{\mathrm{c}}(\mathrm{CO})}\right)^{2 / 3}$ 


\section{Appendix B: List of symbols}

\begin{tabular}{|ll|}
\hline \hline model parameter: \\
\hline$L_{\star}$ & (initial) luminosity of the star \\
$M_{\star}$ & stellar mass \\
$T_{\star}$ & (initial) effective temperature \\
$P$ & period of the stellar pulsation \\
$\Delta u$ & velocity amplitude of the stellar pulsation \\
$\mathrm{C} / \mathrm{O}$ & carbon-to-oxygen element ratio \\
\hline \hline hydro- and thermodynamic quantities: \\
\hline$t$ & time since the onset the calculation \\
$r$ & radial position of a Langrangian mass element \\
$r_{0}$ & initial radial position at $t=0$ \\
$u$ & hydrodynamic velocity \\
$\mathcal{F}_{\text {vis }}$ & force per unit mass via articifial viscosity \\
$M(r)$ & enclosed mass inside radius $r$ \\
$\alpha$ & ratio of radiative acceleration to gravitational decelera- \\
& tion $g$ \\
$T_{\mathrm{g}}$ & kinetic gas temperature \\
$\rho$ & mass density \\
$n_{\langle\mathrm{H}\rangle}$ & total hydrogen particle density $\rho \approx 1.4$ amu $n_{\langle\mathrm{H}\rangle}$ \\
$p$ & thermal gas pressure \\
$V$ & specific volume $1 / \rho$ \\
$V_{0}$ & initial specific volume at $t=0$ \\
$e$ & internal energy of the gas \\
$\widehat{Q}_{\text {rad }}$ & net radiative heating rate \\
$\widehat{Q}_{\text {vis }}$ & heating rate via articifial viscosity \\
\hline \hline radiative transfer: \\
\hline$J$ & freq. integrated mean intensity $J=\sigma T_{\text {rad }}^{4} / \pi$ \\
$F$ & frequency integrated radiative flux \\
$T_{\text {rad }}$ & grey radiative equilibrium temperature \\
$\kappa$ & total (gas + dust) grey extinction coefficient \\
$\mu_{r}$ & cosine of the separation angle dividing the solid angle \\
& into domains with intensities $I^{+}(r) / I^{-}(r)$ \\
$r_{\text {inner }}$ & inner boundary of the model \\
$R$ & outer boundary of the model \\
\hline \hline dust quantities: \\
\hline$f(N)$ & dust size distribution function \\
$K_{j}$ & jth dust moment $K_{j}=\frac{1}{\left.n_{\langle\mathrm{H}\rangle}\right\rangle} \int_{N_{\mathrm{I}}}^{\infty} f(N) N^{j / 3} \mathrm{~d} N$ \\
$N$ & number of carbon atoms in a dust grain \\
$N_{\mathrm{l}}$ & minimum size of dust grains included in $K_{j}$ \\
$J\left(N_{\mathrm{l}}\right)$ & flux of particles through the lower integration boundary \\
\hline physical constants: \\
\hline$G$ & $N_{\mathrm{l}}$ \\
\hline
\end{tabular}

\section{References}

Bowen, G. H. 1988, ApJ, 329, 299

Elitzur, M. 1983, ApJ, 266, 609

Fleischer, A. J. 1994, Ph.D. Thesis, Technische Universität, Berlin, Germany

Fleischer, A. J., Gauger, A., \& Sedlmayr, E. 1992, A\&A, 266, 321 Fleischer, A. J., Gauger, A., \& Sedlmayr, E. 1995, A\&A, 297, 543 Gail, H.-P., Keller, R., \& Sedlmayr, E. 1984, A\&A, 133, 320

Gail, H.-P., \& Sedlmayr, E. 1987a, A\&A, 171, 197

Gail, H.-P., \& Sedlmayr, E. 1987b, A\&A, 177, 186

Gail, H.-P., \& Sedlmayr, E. 1988, A\&A, 206, 153
Gauger, A., Gail, H.-P., \& Sedlmayr, E. 1990, A\&A, 235, 345

Habing, H. 1996, A\&AR, 7, 97

Hashimoto, O. 1995, ApJ, 442, 286

Helling, Ch. 1999, Ph.D. Thesis, Technische Universität Berlin, Germany

Helling, Ch., Arndt, T., \& Sedlmayr, E. 2002, in Radial and Nonradial Pulsations as Probes of Stellar Physics, ed. C. Aerts, T. Bedding, \& C. Christensen-Dalsgaard (Michigan: Astronomical Society of the Pacific), IAU Coll., 185, 546

Helling, Ch., Winters, J. M., \& Sedlmayr, E. 2000, A\&A, 358, 651

Höfner, S., Feuchtinger, M. U., \& Dorfi, E. A. 1995, A\&A, 297, 815

Höfner, S., Gautschy-Loidl, R., Aringer, B., \& Jørgensen, U. G. 2003, A\&A, 399, 589

Hollenbach, D., \& McKee, F. 1979, ApJS, 41, 555

Hollenbach, D., \& McKee, F. 1989, ApJ, 342, 306

Hummer, D. G. 1988, ApJ, 327, 477

John, T. L. 1994, MNRAS, 266, 186

John, T. L., \& Williams, R. J. 1977, MNRAS, 181, 483

Lepp, S., \& Shull, M. 1983, ApJ, 270, 578

Maciel, W. J. 1977, A\&A, 57, 273

Mihalas, D. 1978, Stellar Atmospheres, 2nd ed. (San Francisco: W. H. Freeman and Company)

Millikan, R. C., \& White, D. R. 1964, J. Chem. Phys., 39, 3209

Neufeld, D. A., \& Hollenbach, D. J. 1994, ApJ, 428, 170

Olofsson, H., Bergman, P., Lucas, R., et al. 2000, A\&A, 353, 583

Pijpers, F. P., \& Habing, H. J. 1989, A\&A, 215, 334

Pijpers, F. P., \& Hearn, A. G. 1989, A\&A, 209, 198

Puls, J., \& Hummer, D. J. 1988, A\&A, 191, 87

Richter, H., \& Wood, P. R. 2001, A\&A, 369, 1027

Richter, H., Wood, P. R., Woitke, P., Bolick, U., \& Sedlmayr, E. 2002, A\&A, submitted

Richtmyer, R. D., \& Morton, K. W. 1967, Difference methods for initial-value problems, 2nd edn. (New York: John Wiley \& Sons)

Rothman, L. S., Gamache, R. R., Goldman, A., et al. 1987, Appl. Opt., 26,4058

Somerville, W. B. 1964, ApJ, 139, 192

Stilley, J. L., \& Callaway, J. 1970, ApJ, 160, 245

Tscharnuter, W. M., \& Winkler, K.-H. 1979, Comp. Phys. Comm., 18, 171

Tsuji, T., Ohnaka, K., Aoki, W., \& Yamamura, I. 1997, A\&A, 320, L1

Turner, J., Kirby-Docken, K., \& Dalgarno, A. 1977, ApJS, 35, 281

Unno, W., \& Kondo, M. 1976, PASJ, 28, 347

Wachter, A. C., Schröder, K.-P., Winters, J. M., Arndt, T. U., \& Sedlmayr, E. 2002, A\&A, 384, 452

Wallerstein, G., \& Knapp, G. R. 1998, ARA\&A, 36, 369

Willson, L. A. 2000, ARA\&A, 38, 573

Willson, L. A., \& Bowen, G. H. 1998, in Cyclical variability in stellar winds, ed. L. Kaper, \& A. W. Fullerton (Springer), 294

Winters, J. M., Fleischer, A. J., Le Bertre, T., \& Sedlmayr, E. 1997, A\&A, 326, 305

Winters, J. M., Le Bertre, T., Jeong, K. S., Helling, Ch., \& Sedlmayr, E. 2000, A\&A, 361, 641

Wishart, A. W. 1979, MNRAS, 187, 59p

Woitke, P. 2002, in Modelling of Stellar Atmospheres, ed. N. E. Piskunov, W. W. Weiss, \& D. F. Gray, IAU Symp., 210, in press

Woitke, P., Krüger, D., \& Sedlmayr, E. 1996a, A\&A, 311, 927 (Paper I)

Woitke, P., Goeres, A., \& Sedlmayr, E. 1996b, A\&A, 313, 217 (Paper II)

Woitke, P., \& Schirrmacher, V. 1999, in Abstracts of the 2nd Austrian ISO Workshop. Atmospheres of M, S and C Giants, ed. J. Hron, \& S. Höfner (Institute of Astronomy, University of Vienna), 23

Woitke, P., \& Sedlmayr, E. 1999, A\&A, 347, 617

Wood, P. R. 1979, ApJ, 227, 220 